\title{
On mammalian sperm dimensions
}

\author{
J. M. Cummins and P. F. Woodall \\ Reproductive Biology Group, Department of Veterinary Anatomy, University of Queensland, St Lucia, \\ Queensland 4067, Australia
}

\begin{abstract}
Summary. Data on linear sperm dimensions in mammals are presented. There is information on a total of 284 species, representing $6.2 \%$ of all species; $17 \cdot 2 \%$ of all genera and $49 \cdot 2 \%$ of all families have some representation, with quantitative information missing only from the orders Dermoptera, Pholidota, Sirenia and Tubulidentata. In general, sperm size is inverse to body mass (except for the Chiroptera), so that the smallest known spermatozoa are amongst those of artiodactyls and the largest are amongst those of marsupials. Most variations are due to differences in the lengths of midpiece and principal piece, with head lengths relatively uniform throughout the mammals.
\end{abstract}

\section{Introduction}

There is increasing interest in comparative studies of gametes both from the phylogenetic viewpoint (Afzelius, 1983) and also in the analysis of the evolution of sexual reproduction and anisogamy (Bell, 1982; Parker, 1982). This work emerged as part of a review of the relationship between sperm size and body mass in mammals (Cummins, 1983), in which lack of space precluded the inclusion of raw data. In publishing this catalogue of sperm dimensions we wish to rectify this defect, and to provide a reference point for, and stimulus to, further quantitative work while obviating the need for laborious compilation of raw data. Some aspects of the material presented previously (Cummins, 1983) have been re-analysed in the light of new data.

\section{Materials and Methods}

This catalogue of sperm dimensions has been built up from cited measurements, from personal observations and from communication with other scientists. Where personal measurements are included, they were made on air-dried nigrosin-eosin live/dead sperm smears (Campbell, Dott \& Glover, 1956) using a calibrated projection microscope. Some data are based on measurements made from Retzius' series of monographs (1906-1910). Although Retzius did not, unfortunately, give accurate scales for his illustrations, it appears that in most cases all the spermatozoa on a single plate were drawn to the same scale. On many plates, therefore, it was possible to find a reference spermatozoon of known dimensions, and to estimate the total sperm lengths of other species from this. While these measurements can be no more than rough approximations, they have nevertheless proved useful for comparative work. In Table 1, such measurements are only included when more accurate data for an otherwise unknown group are not available. When it subsequently proved possible to double-check such estimates, they were usually accurate to within $5 \mu \mathrm{m}$.

The weights and classification of eutherian and monotreme mammals are from Walker (1975); marsupial taxonomy is from Kirsch (1977). Species identification is listed as given by the original source. Where necessary, more modern or more widespread synonyms are shown in parentheses. These were obtained from Ellerman (1940, 1941), Hall \& Kelson (1959), Meester \& Setzer (1974), Walker (1975) and Haltenorth \& Diller (1980). 


\section{Results and Discussion}

Table 1 gives a complete listing of all the data that we have collected on sperm dimensions. Excepting only those figures keyed by an asterisk, which are probably inaccurate (see Key to Table 1), all figures have been accepted uncritically, and readers are referred to the original source for details of methodology, ranges and standard errors of mean measurements. Figures given in parentheses are estimates from Retzius' monographs (1906, 1909a, b, c, 1910: see 'Material and Methods'). Table 2 summarizes the extent of our data coverage. We now have collected quantitative data on $6.2 \%$ of all mammalian species, covering $17 \cdot 2 \%$ of genera and $49.2 \%$ of families. We have been unable to find quantitative information for 4 minor orders; the Pholidota (pangolins), the Dermoptera (flying lemurs), the Sirenia (dugongs, manatees) and the Tubulidentata (aardvark). Of the larger orders, as might be expected, the extent of coverage is most comprehensive for those most likely to be used in the laboratory or as domestic species; the Artiodactyla, Perissodactyla, Lagomorpha and Primates. While we have data on more species of rodents (122) than for any other order, because of the enormous diversity and number of species, this represents only $5.3 \%$ of the total-slightly less than the average cover for all mammalian species. Marsupials and monotremes are reasonably well represented, reflecting much recent interest in the use of sperm morphology for phylogenetic analysis (see, for example Harding, Woolley, Shorey \& Carrick, 1982). Rather surprisingly, there are no sperm dimensions published for American marsupials, even though details of morphology have been known since the beginning of the century. This account deals only with linear measurements, and not with other quantitative aspects such as mass, volume or density; for a detailed bibliography before 1964, together with an exhaustive discussion of sperm mensuration in terms of volume, mass and projected surface area, together with a discussion of the possible sources of error in measurement, see van Duijn (1975) and van Duijn \& van Voorst (1971). For a review of the genetics of sperm dimensions in mammals see Beatty $(1970,1972,1975)$, Williams, Beatty \& Burgoyne (1970) and Burgoyne (1975). For correlating sperm dimensions with those of eggs, see Hartman (1929).

Table 1. Linear dimensions $(\mu \mathrm{m})$ of mammalian spermatozoa

\begin{tabular}{|c|c|c|c|c|c|c|c|}
\hline \multirow[b]{2}{*}{ Species (common name) } & \multirow[b]{2}{*}{ Source } & \multicolumn{2}{|c|}{ Head } & \multicolumn{2}{|c|}{ Midpiece } & \multirow{2}{*}{$\begin{array}{c}\text { Principal } \\
\text { piece } \\
\text { length }\end{array}$} & \multirow[b]{2}{*}{ Total } \\
\hline & & Length & Width & Length & Width & & \\
\hline \multicolumn{8}{|l|}{ EUTHERIAN MAMMALS } \\
\hline \multirow{2}{*}{\multicolumn{8}{|c|}{$\begin{array}{l}\text { Order Artiodactyla } \\
\text { Family Bovidae }\end{array}$}} \\
\hline & & & & & & & \\
\hline \multicolumn{8}{|l|}{ Aepyceros melampus } \\
\hline \multicolumn{8}{|l|}{ Antilope cervicapra } \\
\hline (blackbuck) & 2 & - & - & - & - & - & $48 \cdot 4^{*}$ \\
\hline \multicolumn{8}{|l|}{ Alcelaphus buselaphus } \\
\hline (hartebeeste) & 1 & $4 \cdot 82$ & 3.06 & $7 \cdot 23$ & - & $36 \cdot 32$ & $48 \cdot 37$ \\
\hline Bos taurus & 1 & 6.77 & $4 \cdot 27$ & $9 \cdot 83$ & - & 36.93 & $53 \cdot 53$ \\
\hline \multirow{4}{*}{ (ox) } & 3 & - & - & - & - & - & $50-80$ \\
\hline & 4 & - & - & - & - & - & $25.01^{*}$ \\
\hline & 61 & $\begin{array}{r}8.79 \\
-9.71\end{array}$ & $\begin{array}{r}4 \cdot 62 \\
-4 \cdot 72\end{array}$ & - & - & - & - \\
\hline & 70 & $10 \cdot 2$ & $5 \cdot 4$ & & & & \\
\hline \multicolumn{8}{|l|}{ Bubalus bubalis } \\
\hline (water buffalo) & 3 & $9 \cdot 45$ & $5 \cdot 08$ & $14 \cdot 19$ & - & $33 \cdot 35$ & 56.99 \\
\hline \multicolumn{8}{|l|}{ Caprahircus } \\
\hline (goat) & 6 & $8 \cdot 27$ & $4 \cdot 25$ & $11 \cdot 38$ & 0.74 & $39 \cdot 75$ & $59 \cdot 39$ \\
\hline \multicolumn{8}{|l|}{ Capra ibex } \\
\hline
\end{tabular}


Table 1, cont.

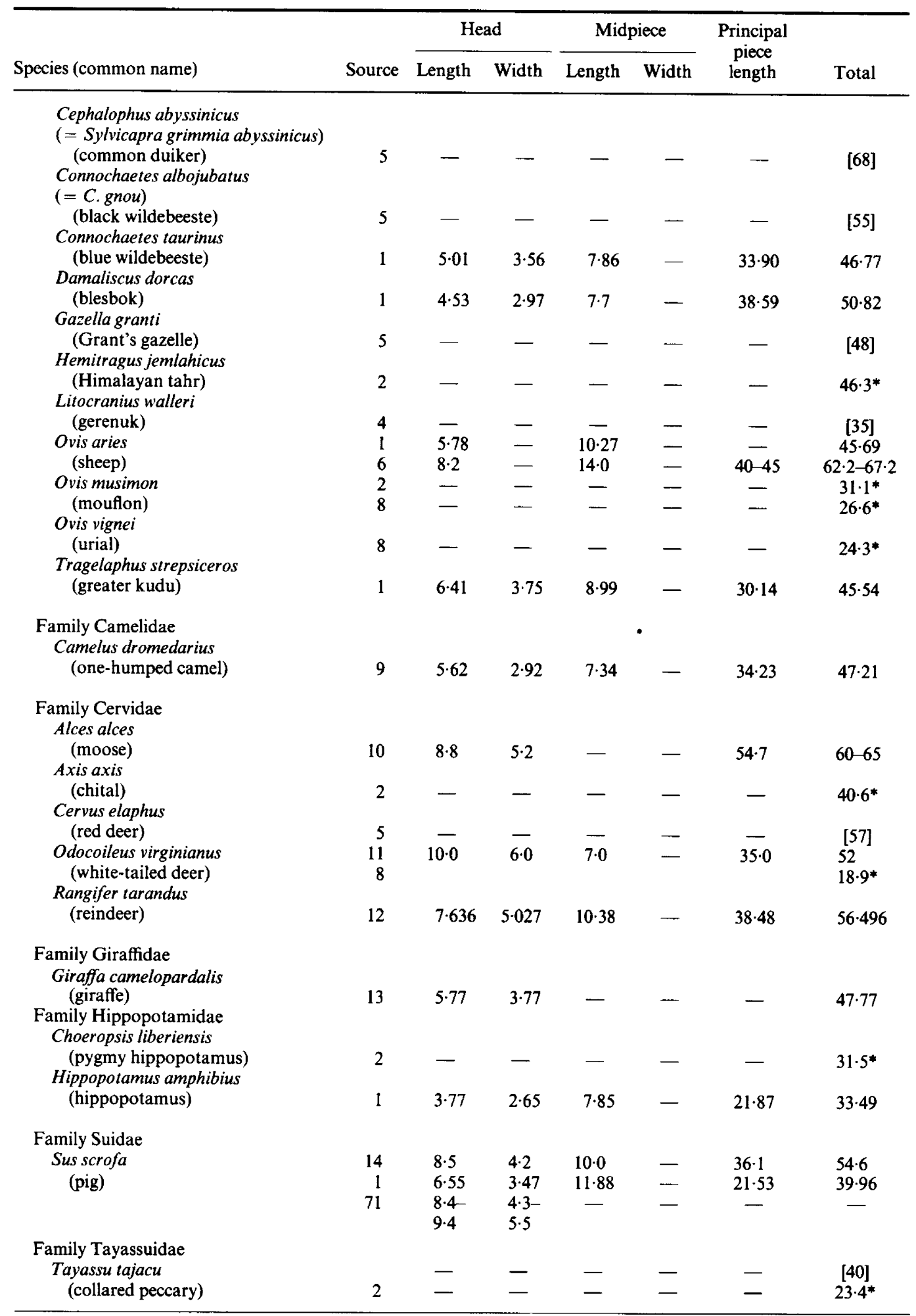


Table 1, cont.

\begin{tabular}{|c|c|c|c|c|c|c|c|}
\hline \multirow[b]{2}{*}{ Species (common name) } & \multirow[b]{2}{*}{ Source } & \multicolumn{2}{|c|}{ Head } & \multicolumn{2}{|c|}{ Midpiece } & \multirow{2}{*}{$\begin{array}{l}\text { Principal } \\
\text { piece } \\
\text { length }\end{array}$} & \multirow[b]{2}{*}{ Total } \\
\hline & & Length & Width & Length & Width & & \\
\hline \multicolumn{8}{|l|}{ Order Carnivora } \\
\hline Canis familiaris & 15 & 5.6 & - & - & - & - & $55 \cdot 3$ \\
\hline$(\operatorname{dog})$ & 4 & - & - & - & - & - & $14 \cdot 54^{*}$ \\
\hline & 67 & 5.9 & 3.9 & $10 \cdot 6$ & - & $45 \cdot 8$ & $62 \cdot 7$ \\
\hline Vulpes fulva & & & & & & & \\
\hline (American red fox) & 16 & 8.0 & $5 \cdot 0$ & - & - & - & $60 \cdot 0$ \\
\hline \multicolumn{8}{|l|}{ Family Felidae } \\
\hline Felis catus & 6 & $4 \cdot 76$ & $2 \cdot 56$ & $8 \cdot 34$ & 0.77 & $46 \cdot 25$ & $59 \cdot 35$ \\
\hline & 4 & - & - & - & - & - & $12 \cdot 92^{*}$ \\
\hline $\begin{array}{l}\text { Panthera tigris } \\
(=\text { Leo tigris) }\end{array}$ & & & & & & & \\
\hline (tiger) & 17 & $5 \cdot 80$ & 3.59 & - & - & - & 43.63 \\
\hline \multicolumn{8}{|l|}{ Family Mustelidae } \\
\hline Meles meles & & & & & & & \\
\hline (badger) & 5 & - & - & - & - & - & [80] \\
\hline $\begin{array}{l}\text { Mustela vison } \\
\quad \text { (mink) }\end{array}$ & 11 & $7 \cdot 0$ & $6 \cdot 1$ & $6 \cdot 0$ & - & $30 \cdot 0$ & $43 \cdot 0$ \\
\hline \multirow{2}{*}{\multicolumn{8}{|c|}{$\begin{array}{l}\text { Family Ursidae } \\
\text { Thalarctos maritimus }\end{array}$}} \\
\hline Thalarctos maritimus & & & & & & & \\
\hline (polar bear) & 8 & - & - & - & - & - & $36 \cdot 3^{*}$ \\
\hline $\begin{array}{l}\text { Ursus arctos } \\
\quad \text { (brown bear) }\end{array}$ & & & & & & & 707 \\
\hline (brown bear) & 2 & - & - & - & - & - & $30 \%$ \\
\hline \multicolumn{8}{|l|}{ Family Viverridae } \\
\hline Cryptoprocta ferox & & & & & & & \\
\hline (fossa) & 5 & - & - & - & - & - & [69] \\
\hline Eupleres goudotii & & & & & & & \\
\hline (small-toothed mongoose) & 5 & - & - & - & - & - & [45] \\
\hline Paradoxurus jerdoni & 2 & & & & & & $\Delta 7 . \Delta^{*}$ \\
\hline & & & & & & & 728 \\
\hline \multicolumn{8}{|l|}{ Family Procyonidae } \\
\hline Ailuropoda melanoleuca & & & & & & & \\
\hline (giant panda) & 18 & $5 \cdot 0$ & $4 \cdot 2$ & $7 \cdot 2$ & $0 \cdot 8$ & $39 \cdot 0$ & $51 \cdot 2$ \\
\hline \multicolumn{8}{|l|}{ Order Cetacea } \\
\hline \multicolumn{8}{|l|}{ Family Balaenopteridae } \\
\hline \multicolumn{8}{|l|}{$\begin{array}{l}\text { Megaptera nodosa } \\
(=M . \text { novaeangliae })\end{array}$} \\
\hline $\begin{array}{r}=\text { M. novaeanglies) } \\
\text { (humpback whale) }\end{array}$ & 19 & - & - & - & - & - & $52 \cdot 5-61 \cdot 4$ \\
\hline \multicolumn{8}{|l|}{ Family Delphinidae } \\
\hline \multirow{2}{*}{\multicolumn{8}{|c|}{$\begin{array}{l}\text { Globicephala melas } \\
(=\text { G. melaena })\end{array}$}} \\
\hline & & & & & & & \\
\hline \multicolumn{8}{|l|}{ Phocoena communis } \\
\hline$(=P$. phocoena $)$ & & & & & & & \\
\hline (common porpoise) & 20 & - & - & - & - & - & $73 \cdot 8$ \\
\hline \multicolumn{8}{|l|}{ Tursiops truncatus } \\
\hline (Atlantic bottle-nosed dolphin) & 21 & $4 \cdot 5$ & $2 \cdot 0$ & $4 \cdot 0$ & - & $56 \cdot 0$ & $64 \cdot 5$ \\
\hline \multicolumn{8}{|l|}{ Family Physeteridae } \\
\hline $\begin{array}{l}\text { Physeter catodon } \\
\text { (sperm whale) }\end{array}$ & 22 & - & - & - & - & - & $45 \cdot 5$ \\
\hline
\end{tabular}


Table 1, cont.

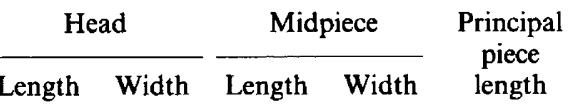

Total

Order Chiroptera

Family Molossidae

Mormopterus planiceps

(= Tadarida planiceps)

(little mastiff-bat)

-

8.0

$52 \cdot 0$

$66 \cdot 0$

Family Phyllostomidae

Anoura cultrata

(long-nosed bat)

Artibeus jamaicensis

(Jamaican fruit-eating bat)

Artibeus lituratus

(big fruit-eating bat)

Carollia castanea

(Allen's short-tailed bat)

Glossophaga soricina

(Pallas' long-tongued bat)

Phyllostomus discolor (pale spear-nosed bat)

Sturnira lilium (yellow-shouldered bat)

Sturnira ludovici

(Anthony's bat)

23

23

23

23

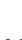

23

23

23

23

Family Pteropodidae

Pteropus alecto

(black flying-fox)

Pteropus conspiculatus (spectacled flying-fox)

Pteropus poliocephalus (grey flying-fox)

Pteropus scapulatus (little red flying-fox)

Pteropus edwardsi (flying fox)

Syconycteris australis (blossom bat)

Family Vespertilionidae

Antrozous pallidus (pallid bat)

Chalinolobus morio (chocolate wattled bat)

Chalinolobus goudii

(Gould's wattled bat)

Eptesicus fuscus

(big brown bat)

Lasionycteris noctivagans

(silver haired bat)

Lasiurus borealis

(red bat)

Miniopterus schreibersii (common bent-wing bat)

Myotis keeni (keen myotis)

Myotis lucifugus (little brown myotis)

Myotis sodalis (Indiana myotis)

73

73

73

6

73

24

73

23

43

43

25

23

23
5.01

$\begin{array}{ll}4 \cdot 35 & 7 \cdot 78\end{array}$

0.76

$50 \cdot 69$

$62 \cdot 58$

$23 \quad 3.80$

3.41

$8 \cdot 28$

$0 \cdot 62$

$4 \cdot 50$

3.45

$9 \cdot 38$

0.96

$71 \cdot 87$

$85 \cdot 75$

$\begin{array}{llll}4.79 & 3.95 & 9.9 & 0.88\end{array}$

$49 \cdot 5$

$64 \cdot 19$

$\begin{array}{lll}4.0 & 3.52 & 7.9\end{array}$

$0 \cdot 96$

5.36

$4 \cdot 18$

9.06

$4.24 \quad 3.8$

$9 \cdot 21$

4.56

3.75

$10 \cdot 46$

0.61

$0 \cdot 82$

0.99

$6 \cdot 2$

$4 \cdot 8$

$22 \cdot 4$

-

$52 \cdot 0$

$2 \cdot 54$

$11 \cdot 95$

$2 \cdot 15$

$43 \cdot 0$

$58 \cdot 54$

$4 \cdot 0$

$-$

6.5

$-$

32-37

$42 \cdot 5-47 \cdot 5$

4.0

-

8-10

$-$

$45 \cdot 0$

57-59

$5 \cdot 0$

9.0

0.8

58.0

$72 \cdot 0$

6.59

2.71

9.89

0.92

$8 \cdot 9$

0.8

$59 \cdot 0$

$73 \cdot 4$

25

$5 \cdot 3$

$2 \cdot 0$

$11 \cdot 2$

0.8

$50 \cdot 6$

$67 \cdot 1$

$43 \quad 9.0$

- 6.0

$$
\text { - }
$$

$50 \cdot 0$

$65 \cdot 0$

$62 \cdot 0$

88.45

$18 \cdot 55$

1.4

$65 \cdot 0$

$51 \cdot 0$ 
Table 1, cont.

\begin{tabular}{|c|c|c|c|c|c|c|c|}
\hline \multirow[b]{2}{*}{ Species (common name) } & \multirow[b]{2}{*}{ Source } & \multicolumn{2}{|c|}{ Head } & \multicolumn{2}{|c|}{ Midpiece } & \multirow{2}{*}{$\begin{array}{l}\text { Principal } \\
\text { piece } \\
\text { length }\end{array}$} & \multirow[b]{2}{*}{ Total } \\
\hline & & Length & Width & Length & Width & & \\
\hline \multicolumn{8}{|l|}{ Myotis austroriparius } \\
\hline $\begin{array}{l}\text { (Mississippi myotis) } \\
\text { Myotis evotis }\end{array}$ & 25 & $4 \cdot 6$ & $1 \cdot 9$ & $12 \cdot 8$ & $1 \cdot 2$ & 31.6 & $49 \cdot 0$ \\
\hline (long-eared myotis) & 23 & $4 \cdot 85$ & $2 \cdot 08$ & $15 \cdot 05$ & 1.33 & - & - \\
\hline $\begin{array}{l}\text { Myotis grisescens } \\
\text { (grey myotis) }\end{array}$ & 23 & 5.81 & 2.53 & 18.6 & & & \\
\hline Myotis nigricans & 23 & 5.01 & 2.35 & 18.6 & 1.83 & $48 \cdot 14$ & 72.55 \\
\hline $\begin{array}{l}\text { (black myotis) } \\
\text { Myotis velifer }\end{array}$ & 23 & $4 \cdot 67$ & $2 \cdot 22$ & $17 \cdot 62$ & $1 \cdot 34$ & - & - \\
\hline $\begin{array}{l}\text { (cave myotis) } \\
\text { Myotis volans }\end{array}$ & 23 & 3.95 & 1.84 & $16 \cdot 55$ & $1 \cdot 70$ & - & - \\
\hline $\begin{array}{l}\text { (long-legged myotis) } \\
\text { Nycticeius humeralis }\end{array}$ & 23 & $5 \cdot 30$ & $2 \cdot 02$ & 15.89 & $1 \cdot 12$ & - & - \\
\hline $\begin{array}{c}\text { (evening bat) } \\
\text { Nyctophilus geoffroyi }\end{array}$ & 25 & $5 \cdot 2$ & $2 \cdot 1$ & $10 \cdot 8$ & $2 \cdot 1$ & $61 \cdot 3$ & $77 \cdot 3$ \\
\hline $\begin{array}{l}\text { (lesser long-eared bat) } \\
\text { Nyctophilus timoriensis }\end{array}$ & 43 & $4 \cdot 0$ & - & $6 \cdot 0$ & - & $43 \cdot 0$ & $53 \cdot 0$ \\
\hline (greater long-eared bat) & 43 & 4.0 & - & 8.0 & - & $40 \cdot 0$ & $52 \cdot 0$ \\
\hline $\begin{array}{l}\text { Pipistrellus subflavus } \\
\text { (eastern pipistrelle) }\end{array}$ & $\begin{array}{l}25 \\
23\end{array}$ & $\begin{array}{l}5 \cdot 4 \\
5 \cdot 81\end{array}$ & $\begin{array}{l}2 \cdot 0 \\
2 \cdot 50\end{array}$ & $\overline{10 \cdot 35}$ & $\begin{array}{l}0.8 \\
0.82\end{array}$ & $7 \overline{70.0}$ & $\begin{array}{l}72 \cdot 0 \\
86 \cdot 16\end{array}$ \\
\hline $\begin{array}{l}\text { Pipistrellus tasmaniensis } \\
\quad \text { (great pipistrelle) }\end{array}$ & 43 & $6 \cdot 0$ & - & 8.0 & - & $52 \cdot 0$ & $66 \cdot 0$ \\
\hline $\begin{array}{l}\text { Plecotus rafinesquii } \\
\text { (eastern big-eared bat) }\end{array}$ & $\begin{array}{l}23 \\
25\end{array}$ & $\begin{array}{l}4 \cdot 74 \\
3 \cdot 8\end{array}$ & $\begin{array}{l}2.25 \\
1.9\end{array}$ & $\begin{array}{l}9 \cdot 59 \\
8 \cdot 9\end{array}$ & $\begin{array}{l}1.41 \\
1 \cdot 1\end{array}$ & $\begin{array}{l}45 \cdot 0 \\
37 \cdot 3\end{array}$ & $\begin{array}{l}59 \cdot 33 \\
50 \cdot 0\end{array}$ \\
\hline $\begin{array}{l}\text { Plecotus townsendii } \\
\quad \text { (western big-eared bat) }\end{array}$ & 23 & $5 \cdot 56$ & 2.79 & $12 \cdot 60$ & 1.52 & - & - \\
\hline
\end{tabular}

\section{Order Edentata}

Family Bradypodidae

Bradypus cuculliger

(= B. tridactylus)

(three-toed sloth)

Family Dasypodidae

Dasypus novemcinctus

(nine-banded armadillo)

$\begin{array}{lll}9.0 & 6.0 & 14.5\end{array}$

$14 \cdot 5$

$45 \cdot 0$

$68 \cdot 0$

Order Hyracoidea

Family Procaviidae

Procavia capensis

(Cape rock hyrax)

$\begin{array}{lllll}62 & 11.5 & 5.5 & 12.0 & 1.5\end{array}$

Order Insectivora

Family Erinaceidae

Erinaceus sp.

(hedgehog)

Family Soricidae

Suncus murinus

(Asiatic musk shrew)

$\begin{array}{lllllll}26 & 21.5 & 17.5 & \sim 20 & - & \sim 80 & 121 \cdot 5\end{array}$

Family Talpidae

Talpa europaea (mole)

$-$

$-\quad-$

一

-

[88]

[31]

Family Tenrecidae

Tenrec eucaudatus (tenrec) 
Table 1, cont.

\begin{tabular}{|c|c|c|c|c|c|c|c|}
\hline \multirow[b]{2}{*}{ Species (common name) } & \multirow[b]{2}{*}{ Source } & \multicolumn{2}{|c|}{ Head } & \multicolumn{2}{|c|}{ Midpiece } & \multirow{2}{*}{$\begin{array}{l}\text { Principal } \\
\text { piece } \\
\text { length }\end{array}$} & \multirow[b]{2}{*}{ Total } \\
\hline & & Length & Width & Length & Width & & \\
\hline $\begin{array}{l}\text { Setifer setosus } \\
\quad \text { (large Madagascar 'hedgehog') }\end{array}$ & 5 & - & - & - & - & - & [64] \\
\hline \\
\hline $\begin{array}{l}\text { Family Leporidae } \\
\text { Bunolagus monticularis }\end{array}$ & & & & & & & \\
\hline (bushman hare) & 30 & $6 \cdot 6$ & $3 \cdot 6$ & - & - & - & - \\
\hline $\begin{array}{l}\text { Lepus capensis } \\
\text { (brown hare) }\end{array}$ & 30 & $6 \cdot 5$ & $4 \cdot 0$ & - & - & - & - \\
\hline $\begin{array}{l}\text { Lepus saxatilis } \\
\text { (scrub hare) } \\
\text { Lepus sp. }\end{array}$ & 30 & 6.7 & $3 \cdot 7$ & - & - & - & - \\
\hline (hare) & 2 & - & - & - & - & - & $29 \cdot 57^{*}$ \\
\hline $\begin{array}{l}\text { Pronolagus crassicaudatus } \\
\text { (= P. randensis) } \\
\text { (Natal red hare) }\end{array}$ & $\begin{array}{l}30 \\
30\end{array}$ & $\begin{array}{l}6 \cdot 7 \\
6 \cdot 4\end{array}$ & $\begin{array}{l}3 \cdot 8 \\
3 \cdot 6\end{array}$ & - & - & - & $\overline{-}$ \\
\hline $\begin{array}{l}\text { Pronolagus rupestris } \\
\text { (Smith's red hare) }\end{array}$ & 30 & 6.8 & 3.7 & - & - & - & - \\
\hline Oryctolagus cuniculus & 27 & $8 \cdot 4$ & - & $8 \cdot 0$ & - & $38 \cdot 0$ & $55 \cdot 0$ \\
\hline (rabbit) & $\begin{array}{r}6 \\
51\end{array}$ & $\begin{array}{l}8.51 \\
8.06- \\
8 \cdot 42\end{array}$ & $\begin{array}{l}4.98 \\
4 \cdot 59 \\
4 \cdot 86\end{array}$ & $\begin{array}{l}8 \cdot 81 \\
-\end{array}$ & $\frac{0 \cdot 73}{-}$ & $\begin{array}{c}40 \cdot 7 \\
-\end{array}$ & $\begin{array}{c}58 \cdot 02 \\
-\end{array}$ \\
\hline
\end{tabular}

Order Perissodactyla

Family Equidae

$\begin{array}{lllllllr}\text { Equus asinus } & 28 & 6.9 & 3.96 & 9.9 & - & 47 \cdot 3 & 64 \cdot 1 \\ \quad \text { (donkey) } & 69 & 5.74 & 3.17 & - & - & - & - \\ \begin{array}{l}\text { Equus caballus } \\ \text { (horse) }\end{array} & 28 & 7.0 & 3.9 & 9.8 & - & 43.8 & 60.6 \\ \begin{array}{l}\text { Equus asinus } \\ \text { (hinny) }\end{array} & 69 & 6.41 & 3.00 & - & - & - & - \\ \begin{array}{l}\text { Equus grevyi } \\ \text { (Grevy's zebra) }\end{array} & 69 & 5.63 & 2.88 & - & - & - & - \\ & 5 & - & - & - & - & - & {[50]}\end{array}$

Order Pinnipedia

Family Phocidae

Mirounga leonina

(southern elephant seal)

Halichoerus grypus

(grey seal)

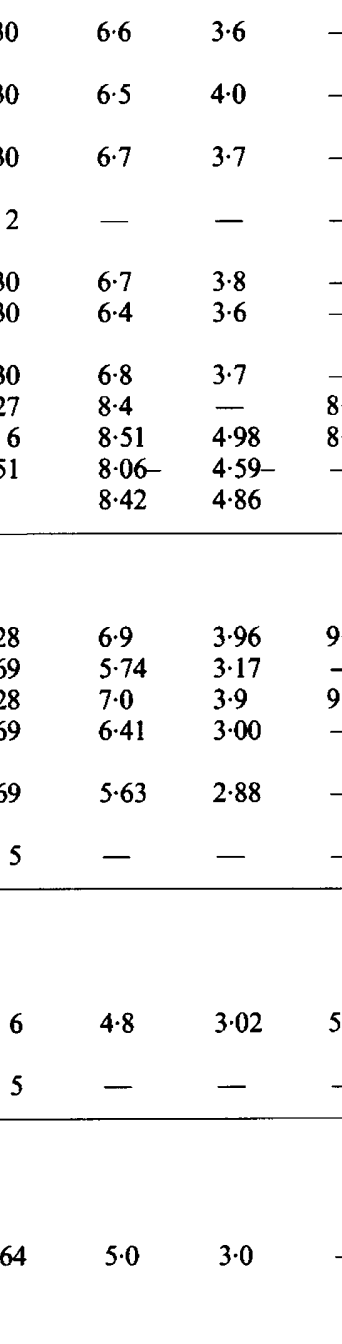

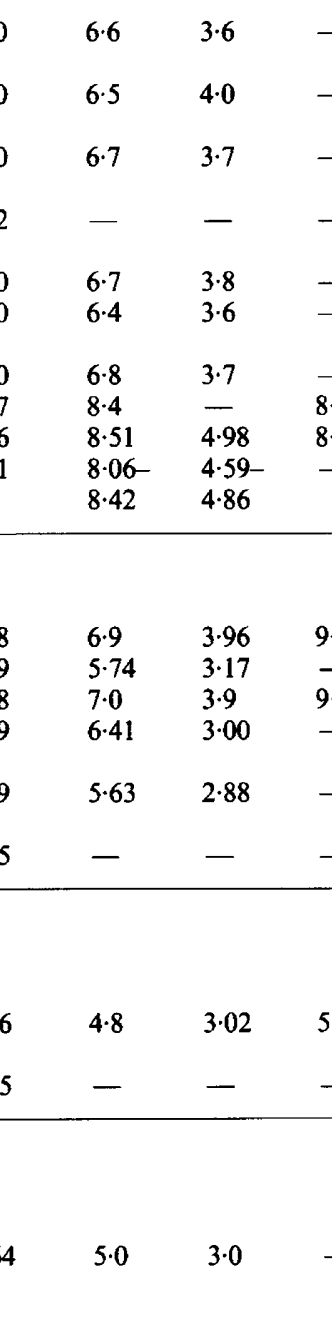

$5 \cdot 3$

0.5

$63 \cdot 6$

$73 \cdot 7$

Order Primates

Family Callitrichidae

Callithrix jacchus

(short-tusked marmoset)

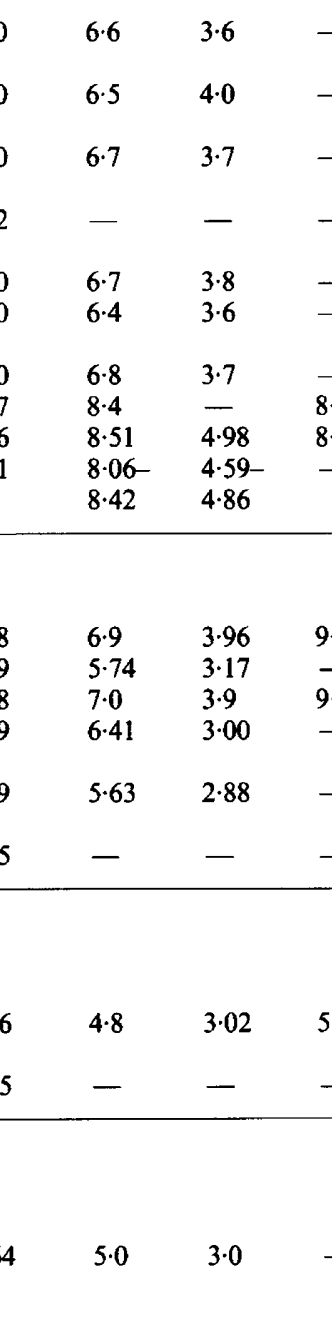

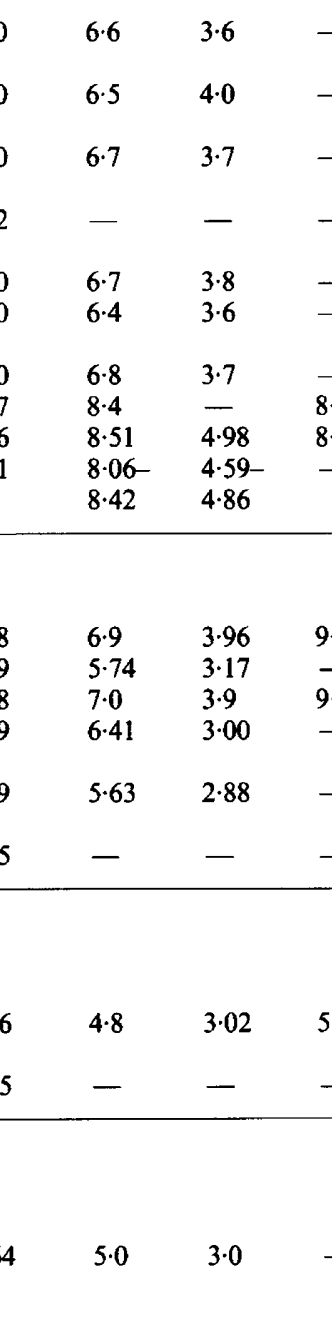

- -

-

50

Family Cebidae

Lagothrix sp.
(woolly monkey)
Saimiri sciureus
(squirrel monkey)

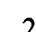

$\begin{array}{lllll}- & - & - & - & 21 \cdot 0^{*} \\ 3.51 & 9.03 & - & 55.34 & 69.24\end{array}$

Family Cercopithecidae

Cercocebus sp.

(mangabey)

Cercopithecus aethiops (vervet monkey)

$\begin{array}{ccccccc}5 & - & - & - & - & - & {[70]} \\ 32 & 5.22 & 3.36 & 10.18 & - & 51.87 & 66.73\end{array}$


Table 1, cont.

\begin{tabular}{|c|c|c|c|c|c|c|c|}
\hline \multirow[b]{2}{*}{ Species (common name) } & \multirow[b]{2}{*}{ Source } & \multicolumn{2}{|c|}{ Head } & \multicolumn{2}{|c|}{ Midpiece } & \multirow{2}{*}{$\begin{array}{l}\text { Principal } \\
\text { piece } \\
\text { length }\end{array}$} & \multirow[b]{2}{*}{ Total } \\
\hline & & Length & Width & Length & Width & & \\
\hline \multicolumn{8}{|l|}{$\begin{array}{l}\text { Cercopithecus fuliginosus } \\
(=\text { Colobus badius temminckii })\end{array}$} \\
\hline $\begin{array}{l}\text { (red colobus) } \\
\text { Cercopithecus pygerythrus }\end{array}$ & 5 & - & - & - & - & - & [84] \\
\hline $\begin{array}{l}\text { (vervet monkey) } \\
\text { Chaeropithecus cynocephalu. } \\
\text { (= Papio cynocephalus) }\end{array}$ & 5 & - & - & - & - & - & [88] \\
\hline $\begin{array}{l}\text { (yellow baboon) } \\
\text { Erythrocebus patas }\end{array}$ & 32 & $4 \cdot 62$ & 3.98 & $10 \cdot 30$ & - & $61 \cdot 06$ & $76 \cdot 19$ \\
\hline $\begin{array}{l}\text { (patas monkey) } \\
\text { Macaca mulatta }\end{array}$ & 2 & - & - & - & - & - & $38 \cdot 7^{*}$ \\
\hline $\begin{array}{l}\text { (rhesus monkey) } \\
\text { Macaca arctoides }\end{array}$ & 32 & $5 \cdot 31$ & $3 \cdot 16$ & $11 \cdot 06$ & - & $57 \cdot 73$ & $73 \cdot 83$ \\
\hline $\begin{array}{l}\text { (stump-tailed macaque) } \\
\text { Macaca fascicularis }\end{array}$ & 32 & $5 \cdot 57$ & $3 \cdot 38$ & $10 \cdot 53$ & - & $61 \cdot 46$ & 77.61 \\
\hline $\begin{array}{l}(=\text { Macaca irus }) \\
\quad \text { (crab-eating macaque) }\end{array}$ & $\begin{array}{r}32 \\
5\end{array}$ & $\begin{array}{l}5 \cdot 53 \\
-\end{array}$ & $\begin{array}{l}3 \cdot 45 \\
-\end{array}$ & $\stackrel{11 \cdot 06}{-}$ & - & $\begin{array}{c}57.04 \\
-\end{array}$ & $\begin{array}{c}73 \cdot 63 \\
{[91]}\end{array}$ \\
\hline \multicolumn{8}{|l|}{$\begin{array}{l}\text { Macaca sinicus } \\
(=M . \text { sinica })\end{array}$} \\
\hline $\begin{array}{l}\text { (toque macaque) } \\
\text { Mandrillus sphinx } \\
\text { (= Papio sphin } x)\end{array}$ & 5 & - & - & - & - & - & {$[68-70]$} \\
\hline (mandrill) & 5 & - & - & - & - & - & {$[67 \cdot 5]$} \\
\hline \multicolumn{8}{|c|}{$\begin{array}{l}\text { Family Daubentoniidae } \\
\text { Chiromys madagascariensis } \\
\text { (= Daubentonia madagascariensis) }\end{array}$} \\
\hline (aye-aye) & 24 & $6 \cdot 3$ & - & - & - & - & $55 \cdot 8$ \\
\hline \multicolumn{8}{|l|}{ Family Hominidae } \\
\hline $\begin{array}{l}\text { Homo sapiens } \\
\quad(\operatorname{man})\end{array}$ & $\begin{array}{l}32 \\
66 \\
72\end{array}$ & $\frac{6 \cdot 11}{4 \cdot 5}$ & $\frac{3 \cdot 45}{3 \cdot 2}$ & $\begin{array}{l}4 \cdot 70 \\
4 \cdot 18 \\
4 \cdot 0\end{array}$ & $\begin{array}{l}\overline{0.88} \\
1.0\end{array}$ & $\begin{array}{l}47 \cdot 13 \\
56 \cdot 16 \\
46-50\end{array}$ & $\begin{array}{c}58.39 \\
- \\
54.5 \\
58.5\end{array}$ \\
\hline & 74 & $4 \cdot 5$ & $3 \cdot 0$ & $5-7$ & - & $50 \cdot 0$ & $\begin{array}{l}59 \cdot 5 \\
61 \cdot 5\end{array}$ \\
\hline \multicolumn{8}{|l|}{$\begin{array}{l}\text { Family Lemuridae } \\
\text { Lemur anjonanensis } \\
(=\text { L. mongoz })\end{array}$} \\
\hline $\begin{array}{l}\text { (mongoose lemur) } \\
\text { Lemur catta }\end{array}$ & 24 & $5 \cdot 8$ & - & - & - & - & $55 \cdot 3$ \\
\hline $\begin{array}{l}\text { (ring-tailed lemur) } \\
\text { Microcebus myoxinus } \\
(=M . \text { murinus })\end{array}$ & 5 & - & - & - & - & - & [52] \\
\hline $\begin{array}{l}\text { (lesser mouse-lemur) } \\
\text { Lepilemur sp. }\end{array}$ & 24 & $8 \cdot 1$ & - & - & - & - & $93 \cdot 6$ \\
\hline (lemur) & 5 & - & - & $\rightarrow$ & - & - & {$[52]$} \\
\hline \multicolumn{8}{|l|}{$\begin{array}{l}\text { Family Pongidae } \\
\text { Gorilla gorilla }\end{array}$} \\
\hline $\begin{array}{l}(\text { gorilla }) \\
\text { Pan troglodytes } \\
(=\text { Chimpansee troglodytes })\end{array}$ & 32 & 4.78 & $7 \cdot 11$ & $11 \cdot 74$ & - & 40.97 & $61 \cdot 17$ \\
\hline $\begin{array}{l}\text { (chimpanzee) } \\
\text { Pongo pygmaeus }\end{array}$ & 32 & $4 \cdot 68$ & $2 \cdot 90$ & $6 \cdot 34$ & - & $46 \cdot 42$ & $57 \cdot 36$ \\
\hline (orangutan) & 32 & $5 \cdot 31$ & $3 \cdot 80$ & $9 \cdot 01$ & - & $52 \cdot 27$ & $66 \cdot 58$ \\
\hline
\end{tabular}


Table 1, cont.

\begin{tabular}{llllllll}
\hline & & \multicolumn{2}{c}{ Head } & & \multicolumn{2}{c}{ Midpiece } & \multicolumn{2}{c}{$\begin{array}{c}\text { Principal } \\
\text { piece }\end{array}$} \\
Species (common name) & Source & Length & Width & Length & Width & length & Total \\
\hline
\end{tabular}

Order Proboscidea

Family Elephantidae

Elephas maximus (Indian elephant)

Loxodonta africana (African elephant)

\begin{tabular}{|c|c|c|c|c|c|c|}
\hline 29 & $8 \cdot 3$ & - & - & - & $42 \cdot 0$ & $50 \cdot 3$ \\
\hline 33 & - & - & - & - & - & 58.5 \\
\hline 65 & $7 \cdot 5$ & 3.0 & $10 \cdot 6$ & - & $40 \cdot 0$ & $58-60$ \\
\hline
\end{tabular}

$\begin{array}{lllllll}34 & - & - & - & - & - & -\end{array}$

Order Rodentia

Family Aplodontidae

Aplodontia rufa (mountain beaver)

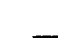

$\begin{array}{rrrcccr}36 & 3.28 & 4.11 & 4.46 & - & - & 34.64 \\ & & & & & & \\ 6 & 10.87 & 9.45 & 11.1 & 1.0 & 92.1 & 114.07 \\ 36 & 9.19 & 10.05 & 8.75 & - & - & 99.15 \\ 36 & 5.45 & 5.68 & 5.89 & - & 32.91 & 44.25\end{array}$

Family Capromyidae Myocastor coypus (coypu)

Family Caviidae

Cavia porcellus (guinea-pig) Galea musteloides (cuis)

$36 \quad 545 \quad 568 \quad 5.89-32.91$

Family Chinchillidae Chinchilla laniger (chinchilla)

Lagidium boxi (mountain viscacha)

\begin{tabular}{|c|c|c|c|c|c|c|}
\hline 36 & 3.76 & $5 \cdot 50$ & 4.73 & - & $38 \cdot 34$ & $46 \cdot 83$ \\
\hline 36 & $3 \cdot 50$ & 5.08 & $5 \cdot 23$ & - & - & $41 \cdot 02$ \\
\hline $\begin{array}{r}37 \\
38 \\
6\end{array}$ & $\begin{array}{l}6 \cdot 85 \\
13 \cdot 2 \\
12 \cdot 66\end{array}$ & $\frac{-}{-}$ & $\begin{array}{l}19.5 \\
105.6 \\
103.06\end{array}$ & - & $\begin{array}{c}59.0 \\
132.2 \\
142.61\end{array}$ & $\begin{array}{c}86 \cdot 7 \\
\sim 250 \\
258 \cdot 32\end{array}$ \\
\hline 5 & $15 \cdot 2$ & $2 \cdot 51$ & 50.5 & $1 \cdot 0$ & 126.3 & 186.8 \\
\hline 39 & $6 \cdot 9$ & - & $27 \cdot 4$ & - & $70 \cdot 0$ & 103.5 \\
\hline 37 & $7 \cdot 6$ & - & $30 \cdot 0$ & - & 80.0 & 117.0 \\
\hline 25 & 7.9 & 4.9 & 21.8 & 0.8 & $64 \cdot 7$ & $94 \cdot 4$ \\
\hline 25 & 4.9 & 3.0 & $21 \cdot 0$ & 0.8 & 49.0 & $74 \cdot 9$ \\
\hline 25 & $8 \cdot 1$ & $1 \cdot 5$ & - & 0.9 & - & $138 \cdot 1$ \\
\hline 37 & 5.39 & - & $16 \cdot 25$ & - & 46.06 & $67 \cdot 7$ \\
\hline 25 & $5 \cdot 5$ & $2 \cdot 1$ & - & 0.9 & - & $79 \cdot 4$ \\
\hline 40 & $5 \cdot 6$ & $2 \cdot 7$ & $18 \cdot 0$ & - & $56 \cdot 0$ & $79 \cdot 6$ \\
\hline 40 & 5.5 & $2 \cdot 4$ & $17 \cdot 0$ & - & $55 \cdot 1$ & $77 \cdot 6$ \\
\hline 40 & $5 \cdot 3$ & 3.4 & $15 \cdot 2$ & - & $51 \cdot 5$ & $72 \cdot 0$ \\
\hline 25 & 5.5 & $3 \cdot 2$ & - & 0.8 & - & $78 \cdot 5$ \\
\hline 40 & $5 \cdot 2$ & $2 \cdot 8$ & $19 \cdot 0$ & - & $54 \cdot 6$ & $78 \cdot 8$ \\
\hline
\end{tabular}

Family Cricetidae Clethrionomys glareolus (bank vole) Cricetulus griseus (Chinese hamster)

Mesocricetus auratus (golden (Syrian) hamster)

Microtus agrestis (field vole)

Microtus hirtus

(= M. agrestis hirtus) (field vole)

Microtus ochrogaster (prairie vole)

Neofiber alleni (eastern wood rat)

Neotoma floridana (Florida water-rat)

Ondatra zibethicus (musk rat)

Oryzomys palustris (rice rat)

Peromyscus banderanus (Michoacan deer mouse)

Peromyscus boylei (brush mouse)

Peromyscus californicus (California mouse)

Peromyscus crinitus (canyon mouse) 
Table 1, cont.

\begin{tabular}{|c|c|c|c|c|c|c|c|}
\hline \multirow[b]{2}{*}{ Species (common name) } & \multirow[b]{2}{*}{ Source } & \multicolumn{2}{|c|}{ Head } & \multicolumn{2}{|c|}{ Midpiece } & \multirow{2}{*}{$\begin{array}{c}\text { Principal } \\
\text { piece } \\
\text { length }\end{array}$} & \multirow[b]{2}{*}{ Total } \\
\hline & & Length & Width & Length & Width & & \\
\hline
\end{tabular}

Peromyscus difficilis (rock mouse)

Peromyscus eremicus (cactus mouse)

Peromyscus flavidus (yellow deer mouse)

Peromyscus floridanus (Florida mouse)

Peromyscus furvus (blackish deer mouse)

Peromyscus gossypinus (cotton mouse)

Peromyscus grandis (big deer mouse)

Peromyscus guatemalensis (Guatemalan deer mouse)

Peromyscus lepturus (slender-tailed deer mouse)

Peromyscus leucopus (white-footed mouse)

Peromyscus lophurus (crested-tailed mouse)

Peromyscus maniculatus (deer mouse)

Peromyscus megalops (brown deer mouse)

Peromyscus melanotis (black-eared mouse)

Peromyscus mexicanus (Mexican deer mouse)

Peromyscus nudipes (naked-footed deer mouse)

Peromyscus nuttalli (golden mouse)

Peromyscus pirrensis (Mt. Pirri deer mouse)

Peromyscus polionotus (old field mouse)

Peromyscus thomasi (Thomas' deer mouse)

Peromyscus truei (piñon mouse)

Peromyscus zarhynchus (Chiapan deer mouse)

Sigmodon hispidus (hispid cotton rat)

Synaptomys cooperi (southern bog lemming)

Tylomys gymnurus (naked-tailed climbing rat)

Tylomys panamensis (Panama climbing rat)

Ototylomys phyllotis

(big-eared climbing rat)

Family Ctenomyidae Ctenomys fulvus Ctenomys magellanicus

\begin{tabular}{|c|c|c|c|c|c|c|}
\hline 40 & $5 \cdot 4$ & $2 \cdot 6$ & 17.0 & - & $56 \cdot 4$ & $78 \cdot 8$ \\
\hline 40 & 5.9 & $3 \cdot 5$ & 16.6 & - & $53 \cdot 5$ & $76 \cdot 0$ \\
\hline 40 & 5.9 & $3 \cdot 0$ & $16 \cdot 8$ & - & $61 \cdot 3$ & 84.0 \\
\hline 40 & $5 \cdot 2$ & 2.9 & 15.9 & - & 53.9 & $75 \cdot 0$ \\
\hline 25 & $5 \cdot 4$ & 2.7 & - & 0.8 & - & 79.9 \\
\hline 40 & $5 \cdot 3$ & 2.5 & $17 \cdot 1$ & - & 54.8 & $77 \cdot 2$ \\
\hline 40 & $5 \cdot 4$ & $2 \cdot 9$ & 17.3 & - & $63 \cdot 4$ & $86 \cdot 1$ \\
\hline 40 & $5 \cdot 3$ & $2 \cdot 5$ & 17.8 & - & 56.7 & 79.8 \\
\hline 40 & $5 \cdot 5$ & $2 \cdot 6$ & 18.2 & - & 58.5 & $82 \cdot 2$ \\
\hline 40 & 6.0 & $4 \cdot 1$ & $19 \cdot 3$ & - & 51.8 & $77 \cdot 1$ \\
\hline 40 & $5 \cdot 3$ & 3.4 & $16 \cdot 8$ & - & 52.7 & $74 \cdot 8$ \\
\hline 25 & $5 \cdot 5$ & 3.0 & - & 0.9 & - & $79 \cdot 8$ \\
\hline 40 & $6 \cdot 2$ & 4.9 & 19.7 & - & $50 \cdot 8$ & 76.7 \\
\hline 40 & $5 \cdot 2$ & 3.0 & $16 \cdot 7$ & - & $53 \cdot 1$ & 75.0 \\
\hline 40 & $5 \cdot 1$ & $2 \cdot 6$ & 19.5 & - & $69 \cdot 3$ & 93.9 \\
\hline 40 & $5 \cdot 3$ & $3 \cdot 1$ & $16 \cdot 7$ & - & $51 \cdot 3$ & $73 \cdot 3$ \\
\hline 40 & $5 \cdot 5$ & $2 \cdot 6$ & $17 \cdot 4$ & - & $49 \cdot 3$ & $72 \cdot 2$ \\
\hline 40 & 5.5 & $2 \cdot 6$ & $17 \cdot 1$ & - & $56 \cdot 6$ & $79 \cdot 2$ \\
\hline 25 & $4 \cdot 2$ & 3.0 & - & 2.0 & - & 67.7 \\
\hline 40 & 5.8 & 2.9 & 16.9 & - & $62 \cdot 2$ & 84.9 \\
\hline 40 & 5.4 & 3.0 & $15 \cdot 2$ & - & $54 \cdot 2$ & 74.8 \\
\hline 25 & 5.4 & $3 \cdot 1$ & - & 1.4 & - & $77 \cdot 4$ \\
\hline 40 & 5.7 & $3 \cdot 1$ & $21 \cdot 4$ & - & $62 \cdot 9$ & $90 \cdot 0$ \\
\hline 40 & $5 \cdot 4$ & $2 \cdot 6$ & $16 \cdot 2$ & - & $56 \cdot 8$ & $78 \cdot 4$ \\
\hline 40 & $5 \cdot 2$ & 2.5 & $17 \cdot 7$ & - & 52.5 & $75 \cdot 4$ \\
\hline 25 & $6 \cdot 1$ & $3 \cdot 2$ & $17 \cdot 2$ & 0.9 & $63 \cdot 1$ & 87.0 \\
\hline 27 & 7.0 & - & $19 \cdot 0$ & - & $60 \cdot 0$ & 87.0 \\
\hline 25 & $7 \cdot 7$ & $4 \cdot 0$ & - & 0.7 & - & 99.7 \\
\hline 41 & 4.86 & 1.6 & 37.52 & - & - & $\sim 87$ \\
\hline 41 & $5 \cdot 47$ & 1.6 & 33.89 & - & - & $\sim 87$ \\
\hline 41 & $5 \cdot 47$ & $2 \cdot 4$ & $94 \cdot 20$ & - & - & $\sim 137$ \\
\hline
\end{tabular}

$78 \cdot 8$

$6 \cdot 0$

$5 \cdot 0$

79.9

$77 \cdot 2$

$86 \cdot 1$

79.8

$82 \cdot 2$

$77 \cdot 1$

$74 \cdot 8$

$76 \cdot 7$

$5 \cdot 0$

93.9

$73 \cdot 3$

$72 \cdot 2$

$79 \cdot 2$

67.7

84.9

$74 \cdot 8$

$75 \cdot 4$

87.0

$87 \cdot 0$

$99 \cdot 7$ 
Table 1, cont.

\begin{tabular}{llllllll}
\hline & & \multicolumn{2}{c}{ Head } & & Midpiece & \multicolumn{2}{c}{$\begin{array}{c}\text { Principal } \\
\text { piece }\end{array}$} \\
Species (common name) & Source & Length & Width & Length & Width & length & Total \\
\hline
\end{tabular}

Ctenomys maulinus
Ctenomys opimus
Ctenomys robustus

Ctenomys talarum

(all known as tuco-tucos)

Family Dasyproctidae

Myoprocta pratti

(acouchi)

Family Dipodidae

Dipus aegyptius

(= Jaculus orientalis $)$

(desert jerboa)

Family Echimyidae

Proechimys guairae

(casiragua)

Family Geomyidae

Geomys pinetus

(south-eastern pocket gopher)

Family Gliridae ( $=$ Myoxidae)

$$
\begin{aligned}
& \text { Glis glis } \\
& \text { (= Myoxus glis) } \\
& \text { (fat dormouse) }
\end{aligned}
$$

Family Heteromyidae

Liomys adspersus

(Panama spiny pocket mouse)

Liomys irroratus

(Mexican pocket mouse)

Liomys pictus

(painted spiny pocket mouse)

Liomys salvini

(Salvin's spiny pocket mouse)

Liomys spectabilis

(spectacled spiny pocket mouse)

Family Muridae

Aethomys chrysophilus

(red veldt rat)

Apodemus flavicollis

(yellow-necked mouse)

Apodemus sylvaticus

(wood mouse)

Berylmys bowersii

(= Rattus bowersii)

(Bower's rat)

Chiropodomys gliroides (brush-tailed rabbit rat)

Conilurus penicillatus (pencil-tailed tree mouse)

Hapolomys longicaudatus (marmoset mouse)

Hydromys chrysogaster (water rat)

$\begin{array}{llllllc}42 & 10.5 & 5.5 & - & - & - & 85.7 \\ 68 & 10.35 & 5.64 & 8.38 & - & - & - \\ 68 & 7.70 & 3.90 & 6.13 & - & - & - \\ 68 & 7.10 & 3.79 & 6.14 & - & - & - \\ 36 & 4.27 & 6.83 & 6.18 & - & 38.83 & 49.28\end{array}$

$\begin{array}{lllllll}36 & 3.05 & 5.17 & 5.62 & - & 31.52 & 40.19\end{array}$

$\begin{array}{lllllll}5 & - & - & - & - & - & \end{array}$

$\begin{array}{lllllll}36 & 3.21 & 5.08 & 4.21 & - & 29.97 & 37.38\end{array}$

$\begin{array}{lllllll}25 & 4.5 & 2.2 & - & 1.1 & - & 110.5\end{array}$

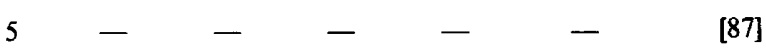

$\begin{array}{lllllll}63 & 3.17 & 2.80 & - & - & - & - \\ 63 & 5.35 & 1.97 & - & - & - & - \\ 63 & 5.22 & 1.81 & - & - & - & - \\ 63 & 3.22 & 2.97 & - & - & - & - \\ 63 & 5.56 & 2.07 & - & - & - & -\end{array}$

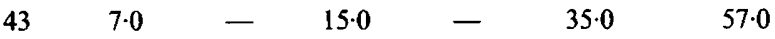

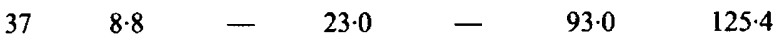

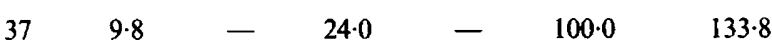

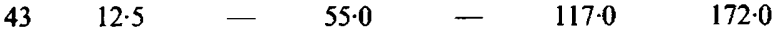

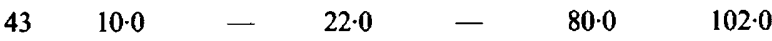

$44 \quad 7.0 \quad-\quad 20-22 \quad-\quad 96 \cdot 0 \quad 123-125$

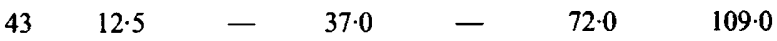

$44 \quad 7.0 \quad-\quad 20.0 \quad-\quad 88.0 \quad 115.0$ 
Table 1, cont.

\begin{tabular}{l}
\hline \\
Species (common name) \\
\hline Leggadina forresti \\
(Forrest's mouse) \\
Leopoldamys sabanus \\
(= Rattus sabanus) \\
(long-tailed giant rat) \\
Leopoldamys edwardsi \\
(= Rattus edwardsi) \\
(Edward's long-tailed giant rat) \\
Leporillus conditor \\
(greater stick-nest rat) \\
Mastomys coucha \\
(= Rattus/Praomys coucha) \\
(multimammate mouse) \\
Mastomys natalensis \\
(= Rattus/Pramys natalensis) \\
(multimammate mouse) \\
Maxomys inas \\
(= Rattus inas) \\
(Malayan mountain spiny rat) \\
Maxomys surifer \\
(= Rattus surifer) \\
(spiny rat) \\
Maxomys whiteheadi \\
(= Rattus whiteheadi) \\
(Whitehead's spiny rat) \\
Melomys littoralis \\
(= M. burtoni) \\
(grassland melomys) \\
Micromys minutus \\
(harvest mouse) \\
Mus musculus \\
(house mouse) \\
\end{tabular}

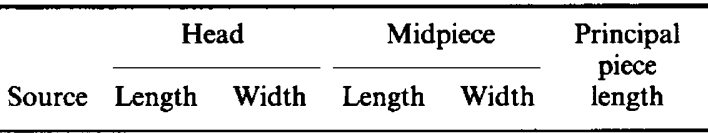

Total

Leggadina forresti

(Forrest's mouse)

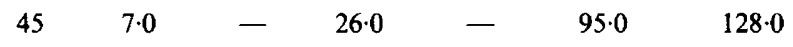

$43 \quad 11.5-67.0-103.0 \quad 170.0$

$\begin{array}{llllllll}43 & 12.5 & - & - & - & - & & 172.0\end{array}$

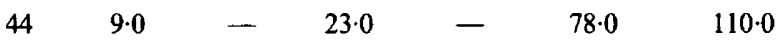

$\begin{array}{lllllll}43 & 10.0 & - & 42.0 & - & 105.0 & 157.0\end{array}$

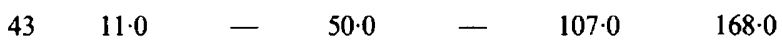

$\begin{array}{lllllll}43 & 13.0 & - & 13.0 & - & 107.0 & 139.0\end{array}$

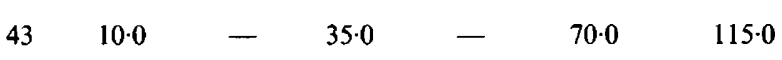

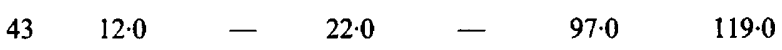

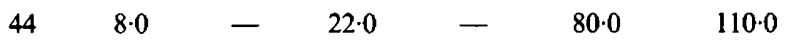

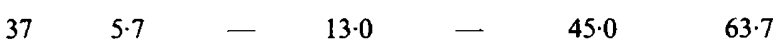

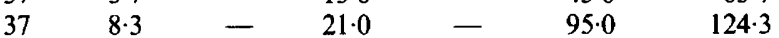

$\begin{array}{lllllll}25 & 7.9 & 3.2 & 18.4 & 1.3 & 96.6 & 122.9\end{array}$

$31 \quad-\begin{array}{lllll}31 & 3.60 & 21.6 & - & -\end{array}$

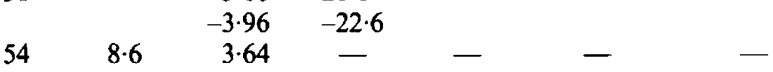

$\begin{array}{rcrcccc}55 & -9.44 & -4.48 & & & & \\ & - & 3.43 & 21.8 & - & - & - \\ & & -3.71 & -22.6 & & & \end{array}$

$\begin{array}{rrrrrr}56 & - & 3.681 & 21.610 & - & -\end{array}$

$\begin{array}{rrrrrrr}57 & 7.24 & 3.26 & 23.34 & - & - & -\end{array}$

$\begin{array}{rrrrrr}58 & 3.639 & 21.76 & - & -\end{array}$

$60-\begin{array}{ccccc}-3.770 & -22.58 & - & -\end{array}$

Niviventer bukit

(= Rattus $n$. bukit)

(white-bellied rat)

Niviventer cremoriventer

( = Rattus n. cremoriventer)

(dark-tailed tree rat)

Niviventer rapit

(= Rattus n. rapit)

(tufted-tailed lesser spiny rat)

Notomys alexis

(spinifex hopping mouse)

Notomys cervinus

(fawn hopping mouse)

$\begin{array}{rrrrrrr}43 & 11.0 & - & 44.0 & - & 97.0 & 152.0 \\ 43 & 9.0 & - & 35.0 & - & 75.0 & 110.0 \\ 43 & 11.0 & - & 46.0 & - & 80.0 & 125.0 \\ 44 & 5-8 & - & 23-27 & - & 70.0 & 100-105 \\ 44 & 9.0 & - & 26.0 & - & 80.0 & 115.0\end{array}$


Table 1, cont.

\begin{tabular}{|c|c|c|c|c|c|c|c|}
\hline \multirow[b]{2}{*}{ Species (common name) } & \multirow[b]{2}{*}{ Source } & \multicolumn{2}{|c|}{ Head } & \multicolumn{2}{|c|}{ Midpiece } & \multirow{2}{*}{$\begin{array}{l}\text { Principal } \\
\text { piece } \\
\text { length }\end{array}$} & \multirow[b]{2}{*}{ Total } \\
\hline & & Length & Width & Length & Width & & \\
\hline $\begin{array}{l}\text { Notomys fuscus } \\
\text { (dusky hopping mouse) }\end{array}$ & 45 & $7 \cdot 0$ & - & 22.0 & - & 77.0 & 106.0 \\
\hline Notomys mitchelli & & 10 & - & & & 年 & 1000 \\
\hline (Mitchell's hopping mouse) & 45 & $9 \cdot 0$ & - & $24 \cdot 0$ & - & $65 \cdot 0$ & $98 \cdot 0$ \\
\hline $\begin{array}{l}\text { Pseudomys apodemoides } \\
\text { (silky mouse) }\end{array}$ & 44 & $8 \cdot 0$ & - & $22 \cdot 0$ & - & $90 \cdot 0$ & $120 \cdot 0$ \\
\hline $\begin{array}{l}\text { Pseudomys australis } \\
\text { (plains rat) }\end{array}$ & 44 & $9 \cdot 0$ & - & $23 \cdot 0$ & - & $88-93$ & $120-125$ \\
\hline $\begin{array}{l}\text { Pseudomys delicatulus } \\
\text { (delicate mouse) }\end{array}$ & 45 & $4 \cdot 0$ & - & $19 \cdot 0$ & - & $65 \cdot 0$ & $88 \cdot 0$ \\
\hline $\begin{array}{l}\text { Pseudomys fumeus } \\
\text { (smokey mouse) }\end{array}$ & 45 & $8 \cdot 0$ & - & $20 \cdot 0$ & - & $100 \cdot 0$ & $128 \cdot 0$ \\
\hline $\begin{array}{l}\text { Pseudomys gracilicaudatus } \\
\text { (eastern chestnut mouse) }\end{array}$ & 45 & $10 \cdot 0$ & - & $21 \cdot 0$ & - & $98 \cdot 0$ & $129 \cdot 0$ \\
\hline $\begin{array}{l}\text { Pseudomys hermannsburgensis } \\
\text { (sandy inland mouse) }\end{array}$ & 44 & $8-10$ & - & $23 \cdot 0$ & - & $85 \cdot 0$ & $116-118$ \\
\hline $\begin{array}{l}\text { Pseudomys higginsi } \\
\text { (long-tailed mouse) } \\
\text { Pseudomys nanus }\end{array}$ & 44 & $8 \cdot 0$ & - & $20-22$ & - & $70-85$ & $98-115$ \\
\hline (western chestnut mouse) & 44 & $9 \cdot 0$ & - & $22 \cdot 0$ & - & $96 \cdot 0$ & $127 \cdot 0$ \\
\hline $\begin{array}{l}\text { Pseudomys novaehollandiae } \\
\text { (New Holland mouse) }\end{array}$ & 45 & $6 \cdot 0$ & - & $22 \cdot 0$ & - & $78 \cdot 0$ & $106 \cdot 0$ \\
\hline $\begin{array}{l}\text { Pseudomys shortridgei } \\
\text { (heath rat) }\end{array}$ & 45 & $5 \cdot 0$ & - & $22 \cdot 0$ & - & $69 \cdot 0$ & $96 \cdot 0$ \\
\hline $\begin{array}{l}\text { Rattus collettl } \\
\text { (dusky rat) } \\
\text { Rattus exulans }\end{array}$ & 44 & $12 \cdot 0$ & - & $51 \cdot 0$ & - & $95 \cdot 0$ & $158 \cdot 0$ \\
\hline $\begin{array}{l}\text { (Polynesian rat) } \\
\text { Rattus fuscipes }\end{array}$ & 43 & $12 \cdot 0$ & - & $67 \cdot 0$ & - & $97 \cdot 0$ & $164 \cdot 0$ \\
\hline $\begin{array}{l}\text { (bush rat) } \\
\text { Rattus leucopus }\end{array}$ & 44 & $12 \cdot 0$ & - & $48 \cdot 0$ & - & $102 \cdot 0$ & $162 \cdot 0$ \\
\hline $\begin{array}{l}\text { (Cape York rat) } \\
\text { Rattus lutreolus }\end{array}$ & 44 & $12 \cdot 0$ & - & - & - & - & $147 \cdot 0$ \\
\hline $\begin{array}{l}\text { (swamp rat) } \\
\text { Rattus norvegicus (white) }\end{array}$ & 44 & $13-15$ & - & $54 \cdot 0$ & - & $95 \cdot 0$ & $162-164$ \\
\hline $\begin{array}{c}\text { (common white rat) } \\
\text { Rattus norvegicus (brown) }\end{array}$ & 37 & $11 \cdot 7$ & - & $67 \cdot 0$ & - & $110 \cdot 0$ & $188 \cdot 7$ \\
\hline (common brown rat) & 37 & $12 \cdot 1$ & - & $67 \cdot 0$ & - & $110 \cdot 0$ & $190 \cdot 1$ \\
\hline Rattus rattus diardii & 37 & $10 \cdot 8$ & $\overline{1.8}$ & $65 \cdot 0$ & $\overline{1 \cdot 0}$ & $\begin{array}{r}90 \cdot 0 \\
-\end{array}$ & $\begin{array}{l}166 \cdot 0 \\
158 \cdot 0\end{array}$ \\
\hline (ship (black) rat) & $\begin{array}{l}25 \\
43\end{array}$ & $\begin{array}{l}11 \cdot 0 \\
10 \cdot 0\end{array}$ & 1.8 & - & 1 & - & $\begin{array}{l}158 \cdot 0 \\
162 \cdot 0\end{array}$ \\
\hline $\begin{array}{l}\text { Rattus sordidus } \\
\quad \text { (canefield rat) }\end{array}$ & 44 & $12 \cdot 0$ & - & $49-50$ & - & $95 \cdot 0$ & $152-157$ \\
\hline $\begin{array}{l}\text { Rattus tiomanicus } \\
\text { (Malaysian wood rat) }\end{array}$ & 43 & 10.0 & - & $67 \cdot 0$ & - & $95 \cdot 0$ & $162 \cdot 0$ \\
\hline $\begin{array}{l}\text { Rhabdomys pumilio } \\
\text { (striped mouse) } \\
\text { Saccostomus campestris }\end{array}$ & 43 & $10 \cdot 0$ & - & $22 \cdot 0$ & - & $85 \cdot 0$ & $117 \cdot 0$ \\
\hline $\begin{array}{l}\text { Saccostomus campestris } \\
\text { (pouched mouse) } \\
\text { Sundamys muelleri } \\
(=\text { Rattus muelleri) }\end{array}$ & 43 & $6 \cdot 0$ & - & $22 \cdot 0$ & - & $115 \cdot 0$ & $143 \cdot 0$ \\
\hline $\begin{array}{c}\text { (Mueller's rat) } \\
\text { Tatera leucogaster }\end{array}$ & 43 & $12 \cdot 5$ & - & $47 \cdot 0$ & - & $100 \cdot 0$ & $150 \cdot 0$ \\
\hline $\begin{array}{l}\text { Tatera leucogaster } \\
\text { (bushveldt gerbil) } \\
\text { Uromys caudimaculatus }\end{array}$ & 43 & $5 \cdot 0$ & - & $30 \cdot 0$ & - & $110 \cdot 0$ & $145 \cdot 0$ \\
\hline $\begin{array}{l}\text { (white-tailed rat) } \\
\text { Zyzomys argurus }\end{array}$ & 44 & $8-10$ & - & $20 \cdot 0$ & - & $72-82$ & $100-112$ \\
\hline (common rock rat) & 44 & $7 \cdot 0$ & - & $22 \cdot 0$ & - & $108 \cdot 0$ & $137 \cdot 0$ \\
\hline
\end{tabular}


Table 1, cont.

\begin{tabular}{|c|c|c|c|c|c|c|c|}
\hline \multirow[b]{2}{*}{ Species (common name) } & \multirow[b]{2}{*}{ Source } & \multicolumn{2}{|c|}{ Head } & \multicolumn{2}{|c|}{ Midpiece } & \multirow{2}{*}{$\begin{array}{l}\text { Principal } \\
\text { piece } \\
\text { length }\end{array}$} & \multirow[b]{2}{*}{ Total } \\
\hline & & Length & Width & Length & Width & & \\
\hline \multicolumn{8}{|l|}{ Family Octodontidae } \\
\hline Octodon degus & 46 & $7 \cdot 7$ & 5.9 & - & - & - & $48 \cdot 7$ \\
\hline (degu) & 36 & $5 \cdot 12$ & 6.83 & 5.90 & - & - & 50.93 \\
\hline \multirow{2}{*}{$\begin{array}{l}\text { Octodontomys gliroides } \\
\text { (choz choz) }\end{array}$} & & & & & & & \\
\hline & 36 & $5 \cdot 29$ & $6 \cdot 50$ & $5 \cdot 32$ & - & $34 \cdot 57$ & $45 \cdot 18$ \\
\hline \multicolumn{8}{|l|}{ Family Sciuridae } \\
\hline \multicolumn{8}{|l|}{ Sciurus niger } \\
\hline (eastern fox squirrel) & 25 & $12 \cdot 1$ & $11 \cdot 6$ & - & 1.9 & - & $122 \cdot 2$ \\
\hline $\begin{array}{l}\text { Sciurus carolinensis } \\
\text { (grey squirrel) }\end{array}$ & 2 & - & - & - & - & - & $27 \cdot 47^{*}$ \\
\hline $\begin{array}{l}\text { Tamiasciurus hudsonicus } \\
\text { (red (spruce) squirrel) }\end{array}$ & 25 & $14 \cdot 0$ & $12 \cdot 2$ & - & $2 \cdot 1$ & - & $131 \cdot 0$ \\
\hline $\begin{array}{l}\text { Family Zapodontidae } \\
\text { Zapus hudsonius } \\
\text { (meadow jumping mouse) }\end{array}$ & 25 & $5 \cdot 1$ & $2 \cdot 9$ & - & 0.8 & - & $76 \cdot 1$ \\
\hline
\end{tabular}

MARSUPIAL MAMMALS

Family Dasyuridae

Dasycercus cristicauda (mulgara)

Dasykulata rosamondae

(= Antechinus rosamondae) (little red antechinus)

Dasyuroides byrnei (kowari)

Dasyurus viverrinus (eastern quoll)

Sarcophilus harrisii

(Tasmanian devil)

\begin{tabular}{|c|c|c|c|c|c|c|}
\hline 50 & 9.5 & 1.9 & - & - & - & - \\
\hline 50 & $9 \cdot 8$ & $1 \cdot 6$ & - & - & - & - \\
\hline 47 & $12 \cdot 7$ & $2 \cdot 5$ & $40 \cdot 7$ & $3 \cdot 1$ & $201 \cdot 4$ & $254 \cdot 8$ \\
\hline 50 & $11 \cdot 0$ & 1.9 & - & - & - & - \\
\hline 47 & $11 \cdot 1$ & $2 \cdot 2$ & $34 \cdot 4$ & $2 \cdot 6$ & 173.4 & $218 \cdot 5$ \\
\hline 6 & 6.67 & $3 \cdot 38$ & $10 \cdot 53$ & $1 \cdot 20$ & $89 \cdot 2$ & $106 \cdot 4$ \\
\hline 47 & $7 \cdot 3$ & $2 \cdot 2$ & $10 \cdot 7$ & $1 \cdot 5$ & $100 \cdot 9$ & 118.9 \\
\hline 50 & $5 \cdot 3$ & 1.4 & - & - & - & - \\
\hline 8 & - & - & - & - & - & $49 \cdot 4^{*}$ \\
\hline 50 & $4 \cdot 5$ & 1.5 & - & - & - & - \\
\hline 47 & $5 \cdot 1$ & $3 \cdot 4$ & $7 \cdot 9$ & $1 \cdot 4$ & 116.0 & 123.9 \\
\hline 50 & $3 \cdot 3$ & $2 \cdot 0$ & - & - & - & - \\
\hline 47 & $7 \cdot 1$ & 1.8 & $11 \cdot 0$ & $1 \cdot 4$ & - & - \\
\hline 6 & $7 \cdot 5$ & $3 \cdot 2$ & $8 \cdot 5$ & $1 \cdot 28$ & 97.0 & $113 \cdot 2$ \\
\hline 6 & $9 \cdot 27$ & 4.89 & $10 \cdot 05$ & 1.43 & $100 \cdot 21$ & $119 \cdot 53$ \\
\hline 53 & $9 \cdot 0$ & $2 \cdot 3$ & $15 \cdot 7$ & $1 \cdot 7$ & - & - \\
\hline 50 & $9 \cdot 1$ & 1.9 & - & - & - & - \\
\hline 47 & $7 \cdot 2$ & $2 \cdot 2$ & $10 \cdot 9$ & $1 \cdot 5$ & $92 \cdot 2$ & $110 \cdot 3$ \\
\hline 6 & $7 \cdot 8$ & 3.05 & $8 \cdot 9$ & 1.45 & $92 \cdot 2$ & $109 \cdot 5$ \\
\hline 47 & 6.0 & $3 \cdot 3$ & $10 \cdot 7$ & 1.8 & 154.4 & $171 \cdot 1$ \\
\hline 50 & $4 \cdot 5$ & $2 \cdot 2$ & - & - & - & - \\
\hline
\end{tabular}

Family Macropodidae

Macropus eugenii

(tammar wallaby)

Macropus cangaru

(= M. giganteus)

(eastern grey kangaroo)

Macropus parma

(parma wallaby)

Macropus robustus (common wallaroo)

Macropus rufus

(= Megaleia rufa)

(red kangaroo)

Macropus agilis

(agile wallaby)

Macropus parryi (whiptail wallaby)

Potorous tridactylus (long-nosed potoroo)

Thylogale stigmatica (red-necked pademelon)

Wallabia bicolor (swamp wallaby)

Family Peramelidae

Isodon macrourus

(northern brown (brindled)

bandicoot) 
Table 1, cont.

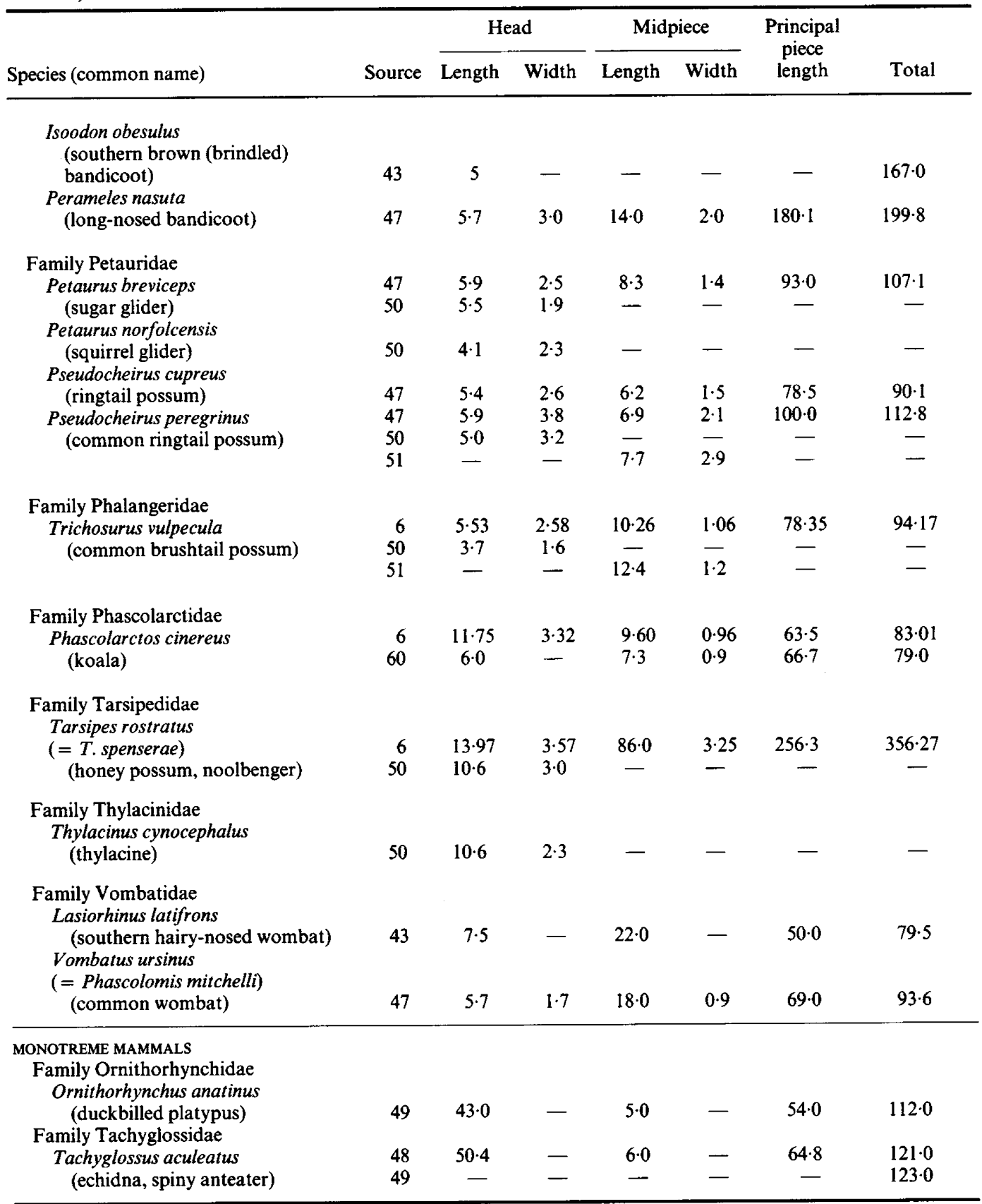

Those lengths given in square brackets are approximations from Retzius' illustrations (see 'Materials and Methods'). Measurements marked with an asterisk are of suspect accuracy, as they come from three papers (Knepp, 1936a,b, 1938 ) in which sperm measurements were made from testicular histology specimens, and several of these are obviously inaccurate (e.g. Felis catus and Canis familiaris). With this exception, all results have been presented uncritically, and the reader is referred to the original source for details of methodology. The midpiece length values for marsupials are measured from the point of insertion on the mid-ventral surface of the head, except for Phascolarctos $(6,60)$. 
References: (1) Morgenthal, 1967; (2) Knepp, 1936b; (3) Venkataswami \& Vedanayagam, 1962; (4) Knepp, 1936a; (5) J. M. Cummins, personal measurements from Retzius' monographs; (6) J. M. Cummins, personal observations; (7) Couturier, 1956; (8) Knepp, 1938; (9) Abdel-Raouf \& El Naggar, 1965; (10) Andersen, 1973; (11) Altman \& Dittmer, 1962; (12) Dott \& Utsi, 1971; (13) Velhankar et al., 1973; (14) Niwa \& Mizuho, 1954; (15) Perez-Garcia, 1957, cited by Asdell, 1964; (16) Beck, 1936; (17) Velhankar et al., 1967; (18) Platz et al., 1983; (19) Chittleborough, 1955; (20) Ballowitz, 1907; (21) Fleming et al., 1981; (22) Yamane, cited by Asdell, 1964; (23) Forman, 1968; (24) Ballowitz, 1909; (25) Hirth, 1960; (26) Green \& Dryden, 1976; (27) Bishop \& Walton, 1960; (28) Nishikawa et al., cited by Asdell, 1964; (29) Asdell, 1964; (30) J. D. Skinner, personal communication; (31) Beatty \& Sharma, 1960; (32) Martin et al., 1975; (33) Jainudeen et al., 1971; (34) Johnson \& Buss, 1967; (35) Pfeiffer, 1956; (36) R. C. Jones, personal communication; (37) Friend, 1936; (38) Bishop \& Walton, 1960: breakdown of component lengths by Cummins from illustration in Austin, 1965; (39) Austin, 1957; (40) Linzey \& Layne, 1974; (41) Helm \& Bowers, 1973; total length of Ototylomys spermatozoon estimated from diagram; (42) Feito \& Gallardo, 1976 (head length does not include the post-acrosomal process); (43) W. G. Breed, personal communication; (44) Breed \& Sarafis, 1979; (45) Breed, 1980; (46) Berrios et al., 1978; (47) Hughes, 1965; (48) Griffiths, 1968; (49) F. N. Carrick, personal communication; (50) Harding et al., 1982: measurements of nucleus alone, from electron microscopy and light microscopy; (51) Harding et al., 1979: measurements by electron microscopy; (52) Beatty \& Napier, 1960; (53) Hughes, 1964; (54) Braden, 1959, range for several strains, measurements in $\mu$ m calculated using given magnification; (55) Sharma, 1960; (56) Pant \& Beatty, 1973; (57) Illison, 1969, measurements in $\mu$ m calculated using given magnification; (58) Burgoyne, 1975; (59) Woolley \& Beatty, 1967; (60) Hughes, 1977; (61) van Duijn \& van Voorst, 1971; (62) J. M. Cummins, personal measurements from illustration in Bedford \& Millar (1978) using given magnification; (63) Genoways, 1973; (64) Davis, 1982; (65) Landowski \& Gill, 1964; (66) Serres et al., 1983 (midpiece length includes neck, and principal piece length includes "terminal filament"); (67) P. F. Woodall, personal observations; (68) Feito \& Gallardo, 1982 (head lengths do not include the post-acrosomal process, where present in C. maulinus and C. magellanicus); (69) Chandley et al., 1974; (70) van Duijn, 1960b; (71) van Duijn, 1960a; (72) van Duijn, 1958 (principal piece includes estimate of 6-10 $\mu \mathrm{m}$ for the "tail end"; (73) S. K. Robson \& G. W. Rouse, personal communication; (74) Flechon \& Hafez, 1976.

While the available information is probably sufficient for us to ask some interesting questions about the evolution of sperm form and function, Table 2 also highlights our ignorance. In particular, it would appear worthwhile to concentrate research on some of the more 'primitive' groups, such as the Insectivora and, amongst the Rodentia, the Sciuridae. The Chiroptera, in particular would be valuable to examine, as flight seems to have imposed certain restrictions on reproductive strategy, and they are anomalous in terms of the inverse sperm length-body mass relationship which seems to hold true for most mammals (Cummins, 1983; and see below).

We have not attempted here to correlate sperm dimensions with any other aspect of gamete morphology such as head shape. For qualitative analyses of mammalian sperm morphology in a wide range of groups the reader is referred to reviews by Bishop \& Austin (1957), Austin \& Bishop (1958), Fawcett (1958, 1970, 1976), Bishop \& Walton (1960), Fawcett, Anderson \& Phillips (1971), Bedford (1974), Jones (1974), Gould, Martin \& Hafez (1975), Matano, Matsubayashi, Omichi \& Ohtomo (1976), Gould (1980) and Elder \& Hsu (1981). In addition, Retzius (1906, 1909a, b, c, 1910) published some beautiful illustrations of spermatozoa of some rare species among, for example, the Carnivora and the Insectivora, which are of undoubted qualitative value in surveying sperm morphology. For a comparative review of sperm morphology in invertebrates as well as vertebrates, see Baccetti \& Afzelius (1976).

Table 3 summarizes the range of sperm dimensions and body masses within mammalian Orders. It is clear that the greatest diversity in sperm length is seen in the Chiroptera, Insectivora, Rodentia and Marsupialia. In Table 4, some of the information previously summarized (Cummins, 1983) has been expanded and reanalysed. Correlations and regressions are based on analysis of $\log _{10}$ total sperm lengths and $\log _{10}$ body masses within mammalian orders, using the median figure for each genus when more than one species is represented. Only data of reasonably good accuracy were used here. In this analysis, in which both variables are subject to error, it is inappropriate to use linear regression. Instead, a Model II regression must be used, calculating the slope of the major or principal axis. When the correlation is reasonably high, the slope of the linear regression $b$ and 
Table 2. Extent of data coverage for mammals

\begin{tabular}{lcccccc}
\hline \multirow{2}{*}{ Order } & \multicolumn{2}{c}{ Families } & \multicolumn{2}{c}{ Genera } & \multicolumn{2}{c}{ Species } \\
\hline Artiodactyla & $7 / 9^{*}$ & $77 \cdot 8 \%$ & $25 / 82^{*}$ & $30 \cdot 2 \%$ & $29 / 197 *$ & $15 \cdot 2 \%$ \\
Carnivora & $6 / 7$ & $85 \cdot 7 \%$ & $12 / 101$ & $11 \cdot 9 \%$ & $12 / 243$ & $4 \cdot 9 \%$ \\
Cetacea & $3 / 8$ & $37 \cdot 5 \%$ & $5 / 38$ & $13 \cdot 2 \%$ & $5 / 90$ & $5 \cdot 6 \%$ \\
Chiroptera & $4 / 18$ & $22 \cdot 2 \%$ & $21 / 180$ & $11 \cdot 7 \%$ & $38 / 804$ & $4 \cdot 7 \%$ \\
Dermoptera & $0 / 1$ & - & $0 / 1$ & - & $0 / 2$ & - \\
Edentata & $2 / 3$ & $66 \cdot 7 \%$ & $2 / 14$ & $14 \cdot 3 \%$ & $2 / 30$ & $6 \cdot 2 \%$ \\
Hyracoidea & $1 / 1$ & $100 \%$ & $1 / 3$ & $33 \cdot 3 \%$ & $1 / 9$ & $11 \cdot 1 \%$ \\
lnsectivora & $4 / 8$ & $50 \cdot 0 \%$ & $5 / 63$ & $7 \cdot 9 \%$ & $5 / \sim 300$ & $1 \cdot 7 \%$ \\
Lagomorpha & $1 / 2$ & $50 \%$ & $4 / 10$ & $40 \cdot 0 \%$ & $8 / 64$ & $12 \cdot 5 \%$ \\
Perissodactyla & $1 / 3$ & $33 \cdot 3 \%$ & $3 / 6$ & $50 \cdot 0 \%$ & $3 / 17$ & $17 \cdot 6 \%$ \\
Pholidota & $0 / 1$ & - & $0 / 1$ & - & $0 / 7$ & - \\
Pinnipedia & $1 / 3$ & $33 \cdot 3 \%$ & $2 / 20$ & $10 \cdot 0 \%$ & $2 / 31$ & $6 \cdot 5 \%$ \\
Primates & $7 / 11$ & $63 \cdot 6 \%$ & $17 / 60$ & $28 \cdot 3 \%$ & $25 / 193$ & $13 \cdot 0 \%$ \\
Proboscidea & $1 / 1$ & $100 \%$ & $2 / 2$ & $100 \%$ & $2 / 2$ & $100 \%$ \\
Rodentia & $16 / 35$ & $45 \cdot 7 \%$ & $55 / 351$ & $15 \cdot 7 \%$ & $122 / \sim 2300$ & $5 \cdot 3 \%$ \\
Sirenia & $0 / 2$ & - & $0 / 2$ & - & $0 / 4$ & - \\
Tubulidentata & $0 / 1$ & - & $0 / 1$ & - & $0 / 1$ & - \\
All Eutheria & $54 / 114$ & $47 \cdot 4 \%$ & $154 / 935$ & $16 \cdot 5 \%$ & $254 / 4294$ & $5 \cdot 9 \%$ \\
\hline All Marsupialia & $9 / 16$ & $56 \cdot 3 \%$ & $19 / 80$ & $23 \cdot 8 \%$ & $28 / 250$ & $10 \cdot 8 \%$ \\
\hline All Monotremata & $2 / 2$ & $100 \%$ & $2 / 3$ & $66 \cdot 7 \%$ & $2 / 6$ & $33 \cdot 3 \%$ \\
\hline All Mammalia & $65 / 132$ & $49 \cdot 2 \%$ & $175 / 1018$ & $17 \cdot 2 \%$ & $284 / 4550$ & $6 \cdot 2 \%$ \\
\hline
\end{tabular}

${ }^{*}$ Number of groups for which some quantitative data exist/total number of groups. Numbers of families, genera and species in each order are from Walker (1975), except for the Marsupialia, where the classification is based on Kirsch (1977).

the slope of the principal axis $\alpha$ will be close in value. It is clear that, in general, total sperm length is inversely correlated with body mass, with the exception of the Chiroptera. As pointed out previously (Cummins, 1983), a negative relationship of this sort is highly unusual. It is not, strictly speaking, negative allometry, in which an organ increases less rapidly than body size when the two are plotted against each other (Clutton-Brock \& Harvey, 1983). The only other commonly cited examples of aspects of body function bearing negative relationships to body mass are metabolic rate and the surface area:volume ratio (Gould, 1966; Clutton-Brock \& Harvey, 1983; SchmidtNeilsen, 1984). Body mass is also inversely related to population density (Damuth, 1981; Peters \& Raelson, 1984), to species density (Peters, 1983) and to the rate of generation turnover in timepresumably in itself related to the capacity of species to adapt to changing or rapidly fluctuating environments (Lindstedt \& Calder, 1981; Fowler \& MacMahon, 1982; Calder, 1983). Thus, some small predators may increase their ability to exploit short-term fluctuations in prey numbers by reducing their generation time (reviewed by Clutton-Brock \& Harvey, 1983).

A negative relationship between sperm size and body mass is by no means universal among mammals, with the Chiroptera being the outstanding exception (Table 4). It is also worth pointing out that, while no 'large' ( $>10 \mathrm{~kg}$ ) mammals have very large spermatozoa, quite clearly the reverse is not necessarily true. A good example of this can be seen in the murid rodent Micromys minutus, which has a mean body weight of only $6 \mathrm{~g}$ and also very small spermatozoa of $63.7 \mu \mathrm{m}$ (Austin, 1957).

In analysing the sperm length-body mass relationship, it would appear that the question to ask is, why do some small mammals, but no large mammals, have very large spermatozoa? In a previous analysis (Cummins, 1983) it was suggested that mammals have the choice between producing 
Table 3. Summary of sperm lengths and body masses in mammals

\begin{tabular}{|c|c|c|c|c|c|c|c|}
\hline \multirow[b]{2}{*}{ Order } & \multirow{2}{*}{$\begin{array}{l}\text { Total } \\
\text { no. of } \\
\text { genera }\end{array}$} & \multirow{2}{*}{$\begin{array}{c}\text { Genera } \\
\text { with } \\
\text { data }\end{array}$} & \multirow{2}{*}{$\begin{array}{l}\text { Species } \\
\text { with } \\
\text { data }\end{array}$} & \multicolumn{2}{|c|}{$\begin{array}{l}\text { Sperm length } \\
(\mu \mathrm{m})\end{array}$} & \multicolumn{2}{|c|}{$\begin{array}{c}\log _{10} \text { body mass } \\
(\mathrm{g})\end{array}$} \\
\hline & & & & Mean* & Range & Mean & Range. \\
\hline Artiodactyla & 82 & 21 & 29 & $50 \cdot 8$ & $33 \cdot 5-80 \cdot 0$ & $5 \cdot 24$ & $4 \cdot 36-6 \cdot 57$ \\
\hline Carnivora & 101 & 9 & 9 & $55 \cdot 2$ & $43 \cdot 3-80 \cdot 0$ & $4 \cdot 18$ & $3 \cdot 20-5 \cdot 40$ \\
\hline Cetacea & 38 & 5 & 5 & $60 \cdot 7$ & $45 \cdot 5-73 \cdot 8$ & $6 \cdot 20$ & $4 \cdot 80-7 \cdot 63$ \\
\hline Chiroptera & 180 & 17 & 28 & $68 \cdot 1$ & $42 \cdot 5-108 \cdot 9$ & $1 \cdot 30$ & $0.78-2.51$ \\
\hline Edentata & 14 & 1 & 1 & $68 \cdot 0$ & - & $3 \cdot 78$ & - \\
\hline Insectivora & 63 & 5 & 5 & $87 \cdot 3$ & $64 \cdot 0-121 \cdot 5$ & $2 \cdot 36$ & $1.65-3.00$ \\
\hline Lägomorpha & 10 & 1 & 1 & $56 \cdot 5$ & - & $3 \cdot 59$ & - \\
\hline Perissodactyla & 6 & 1 & 3 & $62 \cdot 3$ & $50 \cdot 0-64 \cdot 1$ & $5 \cdot 78$ & $4 \cdot 30-6 \cdot 00$ \\
\hline Pinnipedia & 20 & 2 & 2 & $63 \cdot 4$ & $53.0-73.7$ & 5.84 & $5 \cdot 31-6 \cdot 36$ \\
\hline Primates & 60 & 16 & 22 & $65 \cdot 7$ & $50.0-93.6$ & 3.84 & $1 \cdot 80-5 \cdot 32$ \\
\hline Proboscidea & 2 & 2 & 2 & $58 \cdot 4$ & $50 \cdot 3-61 \cdot 6$ & 6.75 & $6 \cdot 70-6 \cdot 80$ \\
\hline Rodentia & 351 & 54 & 113 & $95 \cdot 9$ & $34 \cdot 6-250 \cdot 0$ & $2 \cdot 17$ & $0.78-3.90$ \\
\hline All Eutheria & 935 & 134 & 220 & $73 \cdot 4$ & $33 \cdot 5-250 \cdot 0$ & $3 \cdot 22$ & $0.78-7.63$ \\
\hline All Marsupialia & 80 & 14 & 21 & $133 \cdot 6$ & $79 \cdot 5-356 \cdot 3$ & $3 \cdot 36$ & $1.02-4.57$ \\
\hline All Monotremata & 3 & 2 & 2 & $117 \cdot 0$ & $112 \cdot 0-122 \cdot 0$ & $3 \cdot 48$ & $3 \cdot 23-3 \cdot 72$ \\
\hline All Mammalia & 1018 & 150 & 243 & $78 \cdot 1$ & $33 \cdot 5-356.3$ & $3 \cdot 23$ & $0.78-7.63$ \\
\hline
\end{tabular}

*Means are of median values within genera when more than one species per genus is represented. Data from Knepp (1936a, b; 1938) were not included, as their accuracy is suspect. The numbers differ from those in Table 2 because only species for which total sperm length is known have been included.

Table 4. Correlations of sperm length and body mass in mammals

\begin{tabular}{lrclccc}
\hline & $\mathrm{N}$ & $r$ & & $b$ & $\alpha$ & $95 \%$ limits \\
\hline Artiodactyla & 21 & -0.162 & $P>0.1$ & -0.023 & -0.023 & $+0.037,-0.083$ \\
Carnivora & 9 & -0.154 & $P>0.1$ & -0.021 & -0.021 & $+0.067,-0.109$ \\
Cetacea & 5 & -0.894 & $P<0.05$ & -0.056 & -0.056 & $-0.046,-0.142$ \\
Chiroptera & 19 & +0.640 & $P<0.02$ & +0.125 & +0.128 & $+0.207,+0.051$ \\
Primates & 16 & -0.059 & $P>0.1$ & -0.005 & -0.005 & $+0.037,-0.047$ \\
Rodentia & 54 & -0.301 & $P<0.05$ & -0.101 & -0.113 & $-0.019,-0.209$ \\
\hline $\begin{array}{l}\text { All mammals } \\
\text { Eutherian mammals }\end{array}$ & 149 & -0.445 & $P<0.001$ & -0.054 & -0.555 & $-0.037,-0.073$ \\
Eutheria without & 133 & -0.490 & $P<0.001$ & -0.053 & -0.053 & $-0.037,-0.069$ \\
$\quad \begin{array}{l}\quad \text { Chiroptera } \\
\text { Marsupial mammals }\end{array}$ & 118 & -0.593 & $P<0.001$ & -0.069 & -0.070 & $-0.053,-0.087$ \\
\hline
\end{tabular}

$\mathrm{N}=$ Number of genera for which accurate data on total sperm lengths exist; $r=$ correlation coefficient; $b=$ linear regression; $\alpha=$ principal axis.

few large spermatozoa or many small spermatozoa. In a sense, this argument is based in energetics, supposing that animals tend to invest a relatively constant amount in gamete output. Similar assumptions lie behind most theoretical models concerning the evolution of anisogamy-large sessile female gametes, and small mobile male gametes (Parker, Baker \& Smith, 1972; Charlesworth, 1978; Parker, 1978; Bell, 1978, 1982; Sivinski, 1980; Parker, 1982; Hoekstra, Janz \& Schilstra, 1984; and see also Calow, 1979). Pressures to produce many small spermatozoa are many and diverse, 
not least being inter-male competition in competitive mating situations, and tendencies for spermatozoa to be lost through dispersion in a large female reproductive tract (Parker, 1970, 1982). This may be complicated by other factors such as 'gamete redundancy' (Cohen 1969, 1983; Wallace, 1974) and by variations in breeding system affecting sperm output (Harcourt, Harvey, Larson \& Short, 1981). Cummins (1983) suggested that very large spermatozoa may be selected for when dispersion distances are small, and when circumstances in the female tract favour large, vigorous spermatozoa competing between each other to be first to reach the eggs; while this suggestion obviously needs much more work to validate or to refute, it is supported by at least one theoretical model (G. Bell, personal communication).

If the production of large numbers of small spermatozoa is one way of overcoming tendencies for gametes to be lost by dispersion, then perhaps the anomalous position of the Chiroptera in Table 4 may be explained on the grounds of temporal rather than spatial dispersion. Bats, and particularly small bats of temperate zones, show pronounced capacity for prolonged sperm storage both in the male and the female (Racey, 1975). It would seem to make energetic sense too for chiropterans to invest in many small gametes with limited individual metabolic reserves. Interestingly, the stored spermatozoa appear to enter into a close relationship with the female tract, possibly being nurtured by it (Krutzsch, Crichton \& Nagle, 1982). Chiropteran spermatozoa also possess relatively small midpieces, and midpiece lengths do not correlate closely with overall flagellar length, unlike those of other mammals (see below), again suggesting that bat spermatozoa are unusual in terms of the selection pressures moulding their morphology. Finally, many bats show pronounced female-dominant sexual dimorphism (Ralls, 1976; Myers, 1978), suggesting that spatial dispersion in a large female tract may also be a relatively more important factor than in other mammals of comparable size.

Table 5 summarizes the relative linear proportions of head, midpiece and principal piece for all mammals, and for 5 groups for which there are sufficient numbers represented to warrant analysis. It is apparent that, even though the total length of mammalian spermatozoa varies considerably, the mean head length is relatively constant and it is the other components which exhibit most variation. This is, perhaps, not surprising, as the total amount of nuclear material carried by spermatozoa is fairly constant (van Duijn, 1975). In general, the spermatozoa of rodents and artiodactyls have relatively long midpieces and short principal pieces; those of marsupials are the reverse, with short midpieces, while those for primates and bats are intermediate. While it seems intuitively 'right' that the relative length of the midpiece is a measure of the potential energy output of the spermatozoon, it is difficult to interpret the functional significance of these data without further study.

Further analysis showed that there was a significant positive correlation between the length of the midpiece and the length of the head and principal piece (log values) for all groups except Primates and Chiroptera (Table 6). The changes in midpiece length with increasing total sperm

Table 5. Proportions of component sperm dimensions

\begin{tabular}{|c|c|c|c|c|c|c|}
\hline & \multirow[b]{2}{*}{$\begin{array}{l}\text { No. of } \\
\text { species }\end{array}$} & \multicolumn{2}{|c|}{ Mean length $(\mu \mathrm{m})$} & \multicolumn{3}{|c|}{$\%$ of total length } \\
\hline & & Head & Total & Head & Midpiece & $\begin{array}{l}\text { Principal } \\
\text { piece }\end{array}$ \\
\hline All Mammalia & 171 & $7 \cdot 68$ & $96 \cdot 9$ & $8 \cdot 4$ & $19 \cdot 3$ & $72 \cdot 6$ \\
\hline Artiodactyla & 14 & 6.88 & $52 \cdot 2$ & $13 \cdot 1$ & $19 \cdot 0$ & $67 \cdot 9$ \\
\hline Chiroptera & 23 & $5 \cdot 14$ & $66 \cdot 1$ & 7.9 & $16 \cdot 3$ & $75 \cdot 9$ \\
\hline Primates & 10 & $5 \cdot 22$ & $68 \cdot 1$ & 7.8 & $13 \cdot 7$ & $78 \cdot 0$ \\
\hline Rodentia & 92 & 7.85 & 108.9 & $7 \cdot 4$ & $23 \cdot 2$ & $70 \cdot 3$ \\
\hline Marsupialia & 19 & $7 \cdot 64$ & $140 \cdot 0$ & $6 \cdot 0$ & $11 \cdot 4$ & $82 \cdot 8$ \\
\hline
\end{tabular}


Table 6. Allometry in component sperm dimensions

\begin{tabular}{|c|c|c|c|}
\hline & \multicolumn{3}{|c|}{$\log$ (midpiece length) vs $\log ($ head + principal piece $)$} \\
\hline & $\begin{array}{l}\alpha \text {, slope of principal axis } \\
\text { ( } 95 \% \text { confidence limits) }\end{array}$ & $\begin{array}{l}\text { Correlation } \\
\text { coefficient }(r)\end{array}$ & $\begin{array}{c}\text { Significance } \\
\text { level }\end{array}$ \\
\hline All mammalia & $\begin{array}{c}0.462 \\
(0.379-0.550)\end{array}$ & 0.630 & $P<0.001$ \\
\hline Artiodactyla & $\begin{array}{c}0.361 \\
(0.085-0.694)\end{array}$ & 0.544 & $P<0.05$ \\
\hline Chiroptera & $\begin{array}{c}0 \cdot 183 \\
(-0.223-0.658)\end{array}$ & $0 \cdot 182$ & $P>0.05$ \\
\hline Primates & $\begin{array}{c}0.167 \\
(-0.116-0.478)\end{array}$ & $0 \cdot 341$ & $P>0.05$ \\
\hline Rodentia & $\begin{array}{c}0.491 \\
(0.416-0.571)\end{array}$ & 0.793 & $P<0.001$ \\
\hline Marsupialia & $\begin{array}{c}0.441 \\
(0.205-0.726)\end{array}$ & 0.618 & $P<0.01$ \\
\hline
\end{tabular}

length were not isometric $(\alpha \neq 1)$, but rather showed negative allometry $(\alpha<1: 0 \cdot 36-0 \cdot 49)$, with the midpiece length increasing less rapidly than other components as total sperm length increases. Presumably this is because the changes in dimensions are volumetric rather than simply linear, and the midpiece, being thicker than the principal piece, can be expected to show a low value for $\alpha$ (Gould, 1966).

On a final cautionary note, it is worth re-emphasizing that the data here are presented largely uncritically. The problems of measuring spermatozoa have been reviewed by van Duijn (1975), who points out the many possible errors that can arise either from optical artefact or shrinkage due to drying or fixation. It is difficult to accept, for example, the publication of dimensions with a putative accuracy of $0.001 \mu \mathrm{m}$ when the limit of resolution of the light microscope is in the order of $0.2 \mu \mathrm{m}$.

Thanks are due to all who supplied data, helped in locating material or commented on the work at various stages: in particular B. A. Afzelius, C. R. Austin, J. M. Bedford, W G. Breed, H. M. Dott, B. G. M. Jamieson, R. C. Jones, E. Roldan and J. C. Rodger. Computer storage of the data has been possible due to the facilities provided by the head of this department, Professor T. D. Glover. The authors would be happy to supply updated versions of the data as more information becomes available.

\section{References}

Abdel-Raouf, M. \& El Naggar, M.A. (1965) Reproduction in camels (Camelus dromedarius). 2. The morphology of the camel spermatozoon. J. vet. Sci., U.A.R. 2, 1-11.

Afzelius, B.A. (1983) Sperm structure in relation to function and phylogeny. In The Sperm Cell, pp. 385-394. Ed. J. André. Martinus Nijhoff, The Hague.

Altman, P.L. \& Dittmer, D.S. (1962) Growth, Including Reproduction and Development, p. 163. Federation of American Societies for Experimental Biology, Handbook.

Andersen, K. (1973) Morphological and ultrastructural studies of moose spermatozoa. Acta vet. Scand. 14, $81-91$.
Asdell, S.A. (1964) Patterns of Mammalian Reproduction, 2nd edn. Cornell University Press, Ithaca.

Austin, C.R. (1957) Fertilization, early cleavage and associated phenomena in the field vole (Microtus agrestis). J. Anat. 91, 1-11.

Austin, C.R. (1965) Fertilization, p. 35. Prentice-Hall, Inc., New Jersey.

Austin, C.R. \& Bishop, M.W.H. (1958) Some features of the acrosome and perforatorium in mammalian spermatozoa. Proc. R. Soc. B 149, 234-240.

Baccetti, B. \& Afzelius, B.A. (1976) The Biology of the Sperm Cell. Monographs in Developmental Biology Vol. 10. S. Karger, Basel. 
Ballowitz, E. (1907) Zur kenntnis der spermien der Cetacean. Arch. mikrosk. Anat. EntwMech. 70, 227-237.

Ballowitz, E. (1909) Zur kenntnis der spermien der frugivoren Chiropteren und der Prosimier mit einschluss von Chiromys madagascarensis Desm. Anat. Anz. 34, 275-286.

Beatty, R.A. (1970) The genetics of the mammalian gamete. Biol. Rev. 45, 73-120.

Beatty, R.A. (1972) The genetics of size and shape of spermatozoan organelles. In The Genetics of the Spermatozoon, pp. 95-115. Eds R. A. Beatty \& S. Gluecksohn-Waelsch. Departments of Genetics, University of Edinburgh and Albert Einstein College of Medicine, New York.

Beatty, R.A. (1975) Sperm diversity within the species. In Functional Anatomy of the Spermatozoon, pp. 319-327. Ed. B. A. Afzelius. Pergamon Press, Oxford.

Beatty, R.A. \& Napier, R.A.N. (1960) Genetics of gametes. I. A quantitative analysis of five characteristics of rabbit spermatozoa. Proc. R. Soc. Edinb. B $68,1-16$.

Beatty, R.A. \& Sharma, K.N. (1960) Genetics of gametes. III. Strain differences in spermatozoa from eight inbred strains of mice. Proc. R. Soc. Edinb. B 68, 25-53.

Beck, A. (1936) Dissertation, Leipzig. Cited in Asdell (1964), p. 439.

Bedford, J.M. (1974) Biology of primate spermatozoa. In Reproductive Biology of the Primates, Vol. 3, pp. 97-139. Ed. W. P. Luckett. Karger, Basel.

Bedford, J.M. \& Millar, R.P. (1978) The character of sperm maturation in the epididymis of the ascrotal hyrax, Procavia capensis and armadillo, Dasypus novemcincius. Biol. Reprod. 19, 396-406.

Bell, G. (1978) The evolution of anisogamy. $J$. theor. Biol. 73, 247-270.

Bell, G. (1982) The Masterpiece of Nature. The Evolution and Genetics of Sexuality. Croom Helm, London.

Berrios, M., Flechon, J.E. \& Barros, C. (1978) Ultrastructure of Octodon degus spermatozoon with special reference to the acrosome. Am. J. Anat. 151, 39-54.

Bishop, M.W.H. \& Austin, C.R. (1957) Mammalian spermatozoa. Endeavour 16, 137-150.

Bishop, M.W.H. \& Walton, A. (1960) Spermatogenesis and the structure of mammalian spermatozoa. In Marshall's Physiology of Reproduction, 3rd edn, Vol. 1, part 2, pp. 1-129. Ed. A. S. Parkes. Longmans, Green, London.

Braden, A.W.H. (1959) Strain differences in the morphology of the gametes of the mouse. Aust. J. biol. Sci. 12, 65-71.

Breed, W.G. (1980) Further observations on spermatozoal morphology and male reproductive tract anatomy of Pseudomys and Notomys species. (Mammalia: Rodentia). Trans. Roy. Soc. S. Aust. 104, $51-55$.

Breed, W.G. \& Sarafis, V. (1979) On the phylogenetic significance of spermatozoal morphology and male reproductive anatomy in Australian rodents. Trans. Roy. Soc. S. Aust. 103, 127-135.

Burgoyne, P.S. (1975) Sperm phenotype and its relationship to somatic and germ line genotype: a study using mouse aggregation chimaeras. Devl Biol. 44, 63-76.
Calder, W.A. (1983) Body size, mortality and longevity. $J$. theor. Biol. 102, 135-144.

Calow, P. (1979) The cost of reproduction-a physiological approach. Biol. Rev. 54, 23-40.

Campbell, R.C., Dott, H.M. \& Glover, T.D. (1956) Nigrosin-eosin as a stain for differentiating live and dead spermatozoa. J. agric. Sci., Camb. 48, 1-8.

Chandley, A.C., Jones, R.C., Dott, H.M., Allen, W.R. \& Short, R.V. (1974) Meiosis in interspecific equine hybrids. I. The male mule (Equus asinus $\times$ E. caballus) and hinny (E. caballus $\times E$. asinus). Cytogenet. Cell Genet. 13, 330-341.

Charlesworth, B. (1978) The population genetics of anisogamy. J. theor. Biol. 73, 347-357.

Chittleborough, R.G. (1955) Aspects of reproduction in the male Humpback whale, Megaptera nodosa (Bonaterre). Aust. J. mar. Freshwater Res. 6, 1-29.

Clutton-Brock, T.H. \& Harvey, P.H. (1983) The functional significance of variation in body size among mammals. In Advances in the Study of Animal Behavior, pp. 632-663. Eds J. F. Eisenberg \& D.G. Kleiman. Special Publication No. 7, Am. Soc. Mammalogists, Shippensburg.

Cohen, J. (1969) Why so many sperms? An essay on the arithmetic of reproduction. Sci. Prog., Oxford 57, 23-41.

Cohen, J. (1983) Selection among spermatozoa. In The Sperm Cell, pp. 33-37. Ed. J. André. Martinus Nijhoff, The Hague.

Couturier, M.A.J. (1956) La spermatozoide du Buoquetin des Alpes (Capra ibex ibex L. 1758). Mammalia 20, 124-127.

Cummins, J.M. (1983) Sperm size, body mass and reproduction in mammals. In The Sperm Cell, pp. 395-398. Ed. J. André. Martinus Nijhoff, The Hague.

Damuth, J. (1981) Population density and body size in mammals. Nature, Lond. 290, 699-700.

Davis, M.L. (1982) The spermatozoon of the common Marmoset, Callithrix jacchus (Primates, Callithricidae): an ultrastructural investigation. Ph.D. thesis, Texas A\&M University. [Diss. Abstr. Int. 43B, 1696-1697.]

Dott, H.M. \& Utsi, M.N.P. (1971) The collection and examination of semen of the Reindeer (Rangifer tarandus). J. Zool., Lond. 164, 419-424.

Elder, F.F.B. \& Hsu, T.C. (1981) Silver-staining patterns of mammalian epididymal spermatozoa. Cytogenet. Cell Genet. 30, 157-167.

Ellerman, J.R. (1940, 1941) Families and Genera of Living Rodents, Volumes 1 and 2. British Museum (Natural History), London.

Fawcett, D.W. (1958) The structure of the mammalian spermatozoon. Int. Rev. Cytol. 7, 195-235.

Fawcett, D.W. (1970) A comparative view of sperm ultrastructure. Biol. Reprod., Suppl. 2, 90-127.

Fawcett, D.W. (1976) Unsolved problems in morphogenesis of the mammalian spermatozoon. In International Cell Biology 1976-1977, pp. 588-601. Eds B. R. Brinkley \& K. R. Porter. Rockefeller U. Press, New York.

Fawcett, D.W., Anderson, W.A. \& Phillips, D.M. (1971) Morphogenetic factors influencing the shape of the sperm head. Devl Biol. 26, 220-251.

Feito, R. \& Gallardo, M. (1976) Notes on the sperm 
morphology of Ctenomys maulinus (Rodentia, Octodontidae). Experientia 32, 734-735.

Feito, R. \& Gallardo, M. (1982) Sperm morphology of the Chilean species of Ctenomys (Octodontidae). $J$. Mammal. 63, 658-661.

Flechon, J.E. \& Hafez, E.S.E. (1976) Scanning electron microscopy of human spermatozoa. In Human Semen and Fertility Regulation in Man, pp. 76-82. Ed. E. S. E. Hafez. C. V. Mosby Co., St Louis.

Fleming, A.D., Yanagimachi, R. \& Yanagimachi, H. (1981) Spermatozoa of the Atlantic bottlenosed dolphin, Tursiops truncatus. J. Reprod. Fert. 63, 509-514.

Forman, G.L. (1968) Comparative gross morphology of spermatozoa of two families of North American bats. Univ. Kansas Sci. Bull. 47 (No. 16), 901-908.

Fowler, C.W. \& MacMahon, J.A. (1982) Selective extinction and speciation: their influence on the structure and functioning of communities and ecosystems. Am. Nat. 119, 480-498.

Friend, G.F. (1936) The sperms of the British Muridae. Q. Jl microsc. Sci., N.S. 78, 419-443.

Genoways, H.H. (1973) Systematics and evolutionary relationships of Spiny Pocket Mice, genus Liomys. Special Pubs., Museum Texas Tech. University, No. 5 Texas Tech. Press, Lubbock.

Gould, K.G. (1980) Scanning electron microscopy of the primate sperm. Int. Rev. Cvtol. 63, 323-355.

Gould, K.G., Martin, D.E. \& Hafez, E.S.E. (1975) Mammalian spermatozoa. In SEM atlas of Mammalian Reproduction, pp. 42-57. Ed. E. S. E. Hafez. Springer-Verlag, New York.

Gould, S.J. (1966) Allometry and size in ontogeny and phylogeny. Biol. Rev. 41, 587-640.

Green, J.A. \& Dryden, G.L. (1976) Ultrastructure of epididymal spermatozoa of the Asiatic musk shrew, Suncus murinus. Biol. Reprod. 14, 327-331.

Griffiths, M. (1968) Echidnas. Pergamon Press.

Hall, E.R. \& Kelson, K.R. (1959) The Mammals of North America, Volumes 1 and 2. Ronald Press Co., New York.

Haltenorth, T. \& Diller, H. (1980) A Field Guide to the Mammals of Africa Including Madagascar. Collins, London.

Harcourt, A.H., Harvey, P.H., Larson, S.G. \& Short, R.V. (1981) Testis weight, body weight and breeding system in primates. Nature, Lond. 293, 55-57.

Harding, H.R., Carrick, F.N. \& Shorey, C.D. (1979) Special features of sperm structure and function in marsupials. In The Spermatozoon, pp. 289-303. Eds D. W. Fawcett \& J. M. Bedford. Urban \& Schwarzenberg, Baltimore.

Harding, H.R., Woolley, P.A., Shorey, C.D. \& Carrick, F.N. (1982) Sperm ultrastructure, spermiogenesis and epididymal sperm maturation in Dasyurid marsupials: phylogenetic implications. In Carnivorous Marsupials, pp. 659-673. Ed. M. Archer. Roy. Zool. Soc. New South Wales, Sydney.

Hartman, C.A. (1929) How large is the mammalian egg? A review. Q. Rev. Biol. 4, 373-388.

Hirth, H.F. (1960) The spermatozoa of some North American bats and rodents. J. Morph. 106, 77-83.

Helm, J.D. \& Bowers, J.R. (1973) Spermatozoa of Tylomys and Ototylomys. J. Mammal. 54, 769-772.

Hoekstra, R.F., Janz, R.F. \& Schilstra, A.J. (1984)
Evolution of gamete motility differences. I. Relation between swimming speed and pheromonal attraction. J. theor. Biol. 107, 57-70.

Hughes, R.L. (1964) Sexual development and spermatozoon morphology in the male macropod marsupial Potorous tridactylus (Kerr). Aust. J. Zool. 12, 42-51.

Hughes, R.L. (1965) Comparative morphology of spermatozoa from five marsupial families. Aust. $J$. Zool. 13, 533-543.

Hughes, R.L. (1977) Light and electron microscope studies on the spermatozoa of the koala, Phascolarctos cinereus (Marsupialia). J. Anat. 124, 513.

Illison, L. (1969) Spermatozoal sperm head shape in two inbred strains of mice and their $F 1$ and $F 2$ progenies. Aust. J. Biol. Sci. 22, 947-963.

Jainudeen, M.R., Eisenberg, J.F. \& Jayasinghe, J.B. (1971) Semen of the Ceylon elephant, Elephas maximus. J. Reprod. Fert. 24, 213-217.

Johnson, O.W. \& Buss, I.O. (1967) The testis of the African elephant (Loxodonta africana). J. Reprod. Fert. 13, 11-21.

Jones, R.C. (1974) The ultrastructure of spermatozoa from some hystricomorph rodents. In The Functional Anatomy of the Spermatozoon, pp. 251-258. Ed. B. A. Afzelius. Pergamon Press, Oxford.

Kirsch, J.A.W. (1977) The comparative serology of Marsupialia and a classification of marsupials. Aust. $J$. Zool., Suppl. Ser. 52, 1-152.

Knepp, T.H. (1936a) Comparative study of the testes of the dog, cat, sheep, bull, cottontail rabbit and gray squirrel. Proc. Penn. Acad. Sci. 10, 3942.

Knepp, T.H. (1936b) A quantitative study of the testes of ten mammals. Proc. Penn. Acad. Sci. 10, 58-62.

Knepp, T.H. (1938) A quantitative study of the testes of five mammals. Proc. Penn. Acad. Sci. 12, 61-64.

Krutzsch, P.H., Crichton, E.G. \& Nagle, R.E. (1982) Studies on prolonged spermatozoa survival in Chiroptera. A morphological examination of storage and clearance of intrauterine and cauda epididymal spermatozoa in Myotis lucifugus and M. velifer. Am. J. Anat. 165, 421-434.

Landowski, J. \& Gill, J. (1964) Einige beobachtungen uber das sperma des Indischen Elefanten. (Elephas maximus L.). Zoologischer Garten 29, 205-212.

Lindstedt, S.L. \& Calder, W.A. (1981) Body size, physiological time, and longevity of homeothermic animals. Q. Rev. Biol. 56, 1-16.

Linzey, A.V. \& Layne, J.N. (1974) Comparative morphology of spermatozoa of the Rodent genus Peromyscus (Muridae). Am. Mus. Novit. 2532, 1-20.

Martin, D.E., Gould, K.G. \& Warner, H. (1975) Comparative morphology of primate spermatozoa using scanning electron microscopy. I. Families Hominidae, Pongidae, Cercopithecidae and Cebidae. J. Human Evol. 4, 287-292.

Matano, Y., Matsubayashi, K., Omichi, A. \& Ohtomo, K. (1976) Scanning electron microscopy of mammalian spermatozoa. Gunma Symp. Endocrinology 13, 27-48.

Meester, J. \& Setzer, H.W. (1974) The Mammals of Africa. An Identification Manual. Smithsonian Institute Press, Washington, D.C.

Morgenthal, J.C. (1967) Notes on the spermatozoal morphology of some ungulates. J. S. Afr. vet. med. Ass. 38, 271-273. 
Myers, P. (1978) Sexual dimorphism in size of Vespertilionid bats. Am. Nat. 112, 701-711.

Nishikawa, Y., Waide, Y. \& Onuma, H. (1951) Bull. Nat. Inst. agric. Sci., Chiba, G1; 29-36, 37-45. [Cited in Asdell (1964), pp. 529, 532.]

Niwa, T. \& Mizuho, A. (1954) Bull. Nat. Inst. agric. Sci., Chiba, G8; 31-42. [Cited in Asdell (1964), p. 551.]

Pant, K.P. \& Beatty, R.A. (1973) Post-fertilization maternal effects on sperm dimensions of inbred mouse strains. J. Reprod. Fert. 33, 175-177.

Parker, G.A. (1970) Sperm competition and its evolutionary consequences in the insects. Biol. Rev. 45, 525-567.

Parker, G.A. (1978) Selection on non-random fusion of gametes during the evolution of anisogamy. $J$. theor. Biol. 73, 1-28.

Parker, G.A. (1982) Why are there so many tiny sperm? Sperm competition and the maintenance of two sexes. J. theor. Biol. 96, 281-294.

Parker, G.A., Baker, R.R. \& Smith, V.G.F. (1972) The origin and evolution of gamete dimorphism and the male-female phenomenon. J. theor. Biol. 36, $529-553$.

Perez-Garcia, J. (1957) Rev. Patron. Biol. Anim. (Madrid) 3, 97-150. [Cited in Asdell (1964), p. 433.]

Peters, R.H. (1983) The Ecological Implications of Body Size. Cambridge University Press.

Peters, R.H. \& Raelson, J.V. (1984) Relations between individual size and mammalian population density. Am. Nat. 124, 498-517.

Pfeiffer, E.N. (1956) The male reproductive tract of a primitive rodent, Aplodontia rufa. Anat. Rec. 124, 629-637.

Platz, C.C., Wildt, D.E., Howard, J.G. \& Bush, M. (1983) Electroejaculation and semen analysis and freezing in the giant panda (Ailuropoda melanoleuca). J. Reprod. Fert. 67, 9-12.

Racey, P.A. (1975) The prolonged survival of spermatozoa in bats. In The Biology of the Male Gamete, pp. 385-423. Eds J. G. Duckett \& P. A. Racey. Academic Press, London.

Ralls, K. (1976) Mammals in which females are larger than males. Q. Rev. Biol. 51, 245-276.

Retzius, G. (1906) Spermatozoa of the Marsupialia. Biol. Unters. N.F. 13, 77-86.

Retzius, G. (1909a) Spermatozoa of Didelphys. Biol. Unters. N.F. 14, I23-126.

Retzius, G. (1909b) Spermatozoa of mammals. Biol. Unters. N.F. 14, 163-178.

Retzius, G. (1909c) Spermatozoa of ungulates: Bos taurus. Biol. Unters. N.F. 14, 174.

Retzius, G. (1910) General contribution to the knowledge of spermatozoa with special reference to nuclear material. Biol. Unters. N.F. 15, 63-82.
Schmidt-Neilsen, K. (1984) Scaling: Why is Animal Size so Important? Cambridge University Press.

Serres, C., Escalier, D. \& David, G. (1983) Ultrastructural morphometry of the human sperm flagellum with a stereological analysis of the lengths of the dense fibres. Biol. Cell 49, 153-161.

Sharma, K.N. (1960) Genetics of gametes. IV. The phenotype of mouse spermatozoa in four inbred strains and their Fl crosses. Proc. R. Soc. Edinb. B 68, 54-71.

Sivinski, J. (1980) Sexual selection and insect sperm. $F l$. Ent. 63, 99-111.

van Duijn, C. (1958) Biometry of human spermatozoa. $J$ R. microsc. Soc. 77, 12-27.

van Duijn, C. (1960a) Mensuration of the heads of boar spermatozoa. Mikroskopie 14, 142-156.

van Duijn, C. (1960b) Mensuration of the heads of bull spermatozoa. Mikroskopie 15, 265-276.

van Duijn, C. (1975) Bibliography (with review) on mensuration of spermatozoa. Bibl. Reprod. 25, 121-128, 241-248.

van Duijn, C. \& van Voorst, C. (1971) Precision measurements of dimensions, refractive index and mass of bull spermatozoa in the living state. Mikroskopie 27, 142-167.

Venkataswami, D.P. \& Vedanayagam, A.R. (1962) Biometrics of spermatozoa of cattle and buffaloes. Indian vet. J. 39, 287-291.

Velhankar, D.P., Sane, C.R. \& Kulkarni, M.P. (1967) Spermatozoa of tiger-some observations on their morphology. Indian vet. J. 44, 315-319.

Velhankar, D.P., Hukeri, V.B., Deshpande, B.R. \& Sane, S.R. (1973) Biometry of the genitalia and the spermatozoa of a male giraffe. Indian vet. J. 50, 789-792.

Walker, E.P. (1975) Mammals of the World, 3rd edn, Vols 1 and 2. The John Hopkins Press, Baltimore.

Wallace, H. (1974) Chiasmata have no effect on fertility. Heredity 33, 423-428.

Williams, D.A., Beatty, R.A. \& Burgoyne, P.S. (1970) Multivariate analysis of spermatozoan dimensions in mice. Proc. R. Soc. Lond. B 175, 313-331.

Woolley, D.M., Beatty, R.A. (1967) Inheritance of midpiece length in mouse spermatozoa. Nature, Lond. $215,94-95$.

Yamane, J. (1936) Zeit Zücht. 34B, 105-109. [Cited in Asdell (1964), p. 413.]

Note added in proof: Additional information on 24 species is now available.

Received 22 December 1984 